\title{
SMART TOURISM. UN ESTUDIO DE MAPEO SISTEMÁTICO*
}

\author{
Marco A. Celdrán-Bernabeu ${ }^{a}$ \\ José-Norberto Mazón ${ }^{b}$ \\ Josep A. Ivars-Baidal ${ }^{a}$ \\ J. Fernando Vera-Rebollo ${ }^{a}$
}

Universidad de Alicante

\section{RESUMEN}

El concepto turismo inteligente o "Smart Tourism" se ha convertido en un término de moda ampliamente utilizado por investigadores y profesionales de distintas disciplinas. No obstante, es un concepto que contiene distintos interrogantes que deben ser resueltos para avanzar en el conocimiento científico del tema. Con esta motivación, este artículo contabiliza y categoriza la producción científica asociada, aplicando un estudio de mapeo sistemático, novedoso en el ámbito del turismo. Los resultados obtenidos se combinan para dar respuesta a un conjunto de preguntas de investigación, siguiendo para ello una estrategia o protocolo fundamentado en un proceso sistemático de revisión de la producción científica.

Palabras clave: turismo inteligente; destinos turísticos inteligentes; TIC; mapeo sistemático; datos; gestión turística.

Recibido: 8 de enero de 2018

Devuelto para su revisión: 28 de febrero de 2018

Aceptado: 15 de marzo de 2018

a Instituto Universitario de Investigaciones Turísticas. Universidad de Alicante. E-mail: ma.celdran@ua.es, josep.ivars@ua.es,jf.vera@ua.es

b Instituto Universitario de Investigación Informática. Universidad de Alicante. E-mail: jnmazon@ua.es

El presente trabajo se enmarca en el proyecto de investigación "Análisis de procesos de planificación aplicados a ciudades y destinos turísticos inteligentes. Balance y propuesta metodológica para espacios turísticos: Smart Tourism Planning". Proyecto CSO2017-82592-R del Programa Estatal de I+D+i del Ministerio de Economía, Industria y Competitividad. 


\title{
Smart Tourism. A study on systematic mapping
}

\begin{abstract}
The concept of smart tourism has become a buzzword widely used by researchers and practitioners from different disciplines. However, it is a fuzzy concept that contains different questions that must be resolved in order to advance in his scientific knowledge. With this motivation, this article counts and classifies the associated literature, applying a systematic mapping study, a novel method in the field of tourism. The results obtained are combined to answer a set of research questions, following a strategy or protocol based on a systematic process of literature review.
\end{abstract}

Keywords: Smart tourism; Smart tourism destination; ICT; Systematic mapping; data; tourism management.

\section{INTRODUCCIÓN}

La irrupción de las tecnologías de la información y comunicación (TIC) ha proyectado desde sus orígenes numerosos retos sobre la actividad turística. Como señalan Buhalis y Law (2008), es a comienzos de los años 80 cuando se detecta la poderosa influencia de las TIC en el turismo de la mano de los sistemas globales de distribución (GDS) y las centrales de reserva (CRS).

El desarrollo de Internet a finales de los años 90, puede considerarse como el punto de partida de la actual revolución turístico-tecnológica a la que asiste la industria desde comienzos del S.XXI. Internet es sin duda, la tecnología que más ha influido en el turismo, al haberse convertido en un medio de comunicación universal e interactivo que ha permitido modificar la manera en la que los productos y servicios turísticos son distribuidos (Werthner y Klein,1999). Estos avances en las TIC cambiaron las estrategias de negocio y la estructura de la industria (Porter, 2001). De esta manera, el turista ha cambiado su comportamiento y se ha convertido en el propio gestor de su experiencia, modificando de manera radical el proceso de intermediación turística (Buhalis y Licata, 2002) y pasando de un viajero offline, a un viajero online independiente que se inspira directamente en los e-destinations (Buhalis, 2003; Buhalis, Wagner, y Kingdom, 2013). Para ello utilizan las plataformas Web 2.0 que permiten la interacción con el destino y la co-creación de experiencias (Chung, Lee, Lee y Koo, 2015; Gretzel, Sigala, Xiang, y Koo, 2015; Munar y Jacobsen, 2014; Parra-López, E., ; Bulchand-Gidumal, J., Gutiérrez-Taño, D y DíazArmas, R, 2011; Xiang, Wang, O’Leary, y Fesenmaier, 2015) muy distantes de las antiguas plataformas HTML (HyperText Markup Language) o Web 1.0.

Es por ello que la aparición de Internet y las TIC han facilitado enormemente el nacimiento de un escenario turístico-digital (Jacobsen y Munar, 2012) y el auge de un nuevo turista, hiperconectado y multicanal, que mantiene una estrecha vinculación con los dispositivos móviles y el uso de las tecnologías de la información y comunicación (Zheng Xiang, Magnini, y Fesenmaier, 2015; Buhalis y Foerste, 2014). 
En este escenario dominado por la influencia tecnológica en todos los procesos de innovación turística (Hjalager, 2013), nos encontramos ahora con experiencias renovadas donde el turista hace uso de la tecnología para obtener servicios personalizados (Neuhofer, Buhalis, y Ladkin, 2015) que se adaptan totalmente a sus necesidades y no a los intereses político-administrativos (Buhalis, 2000). Se produce así el empoderamiento del turista gracias al uso de la tecnología (Buhalis, 2011), en todas las fases del viaje, para la cocreación de experiencias online y offline (Neuhofer, Buhalis, y Ladkin, 2012).

Para los destinos turísticos supone un cambio sustancial, fundamentalmente en la forma tradicional de ofrecer y crear servicios y productos. El mayor de los retos es afrontar con éxito el cambio de mentalidad en la gestión de los destinos (Ivars-Baidal, Celdrán-Bernabeu y Vera-Rebollo, 2017), que han pasado a un segundo nivel en la generación de productos y servicios (Buhalis y Foerste, 2014). El turista genera ahora sus propias experiencias gracias a los procesos de co-creación antes, durante y después del viaje(Buhalis y Amaranggana, 2015; Neuhofer, Buhalis, y Ladkin, 2015)que son soportados por las diferentes soluciones tecnológicas (Ej. Redes sociales, apps, videos inspiradores, foros, plataformas de venta online, blogs, etc.). Estas tecnologías han sido el acicate para interesantes modelos de negocio (Gretzel, Werthner, Koo y Lamsfus, 2015), como por ejemplo la denominada economía colaborativa.

Ante estos condicionantes, tanto destinos como empresas turísticas deben ser permeables y modular sus estrategias de actuación mediante la aplicación de enfoques de gestión renovados (Ivars-Baidal, Celdrán-Bernabeu, Mazón, y Perles-Ivars, 2017; Ivars-Baidal, Solsona Monzonís, y Giner Sánchez, 2016); López de Ávila y García, 2015; Luque, Zayas y Caro, 2015).

En medio de esta encrucijada turístico-tecnológica, el presente artículo se propone identificar y caracterizar la producción científica asociada al concepto turismo inteligente o "Smart Tourism" (de aquí en adelante, ST). Una etiqueta de moda que buscamos clarificar en su aplicación al dominio turístico mediante el desarrollo de un estudio de mapeo sistemático de acuerdo a las recomendaciones de Kitchenham, Budgen y Brereton (2011) y Petersen, Feldt, Mujtaba y Mattsson (2008). Mediante esta técnica se clasifican las publicaciones relacionadas, de acuerdo a una serie de preguntas de investigación. Como resultado, se obtiene una caracterización de las publicaciones que ayuda a interpretar brechas en el conocimiento y oportunidades para publicaciones futuras.

Además de la introducción, este artículo consta de cuatro apartados fundamentales. Por una parte, el segundo epígrafe realiza un análisis del estado de la cuestión para conceptualizar el ST y guiar la estrategia de búsqueda. Por otra, el apartado tercero recoge toda la carga metodológica, para en el apartado cuarto, clasificar las investigaciones aportando como valor añadido un mapeo sistemático de la misma. Como colofón, el apartado quinto recoge las conclusiones, posibles limitaciones y orientaciones para investigaciones futuras.

\section{LA APLICACIÓN DEL CONCEPTO SMART AL ÁMBITO TURÍSTICO}

\subsection{La Smart City o Ciudad Inteligente como solución a los problemas urbanos del siglo XXI}

Desde mediados del siglo XX las áreas urbanas han desarrollado un proceso de crecimiento continuado (ONU-HABITAT, 2016) que ha puesto de manifiesto la necesidad de 
establecer límites y proponer modelos urbanos más sostenibles, que den respuesta a los problemas sociales, económicos y ambientales de las ciudades (Moreno, 2015). En este sentido, es importante subrayar el hecho de que más de la mitad de la población vive en zonas urbanas (Naciones Unidas, 2014), lo que implica una urbanización creciente, con diferentes ritmos regionales, que deriva en extraordinarios retos para la planificación y gestión de las ciudades del siglo XXI, fundamentalmente para las megalópolis. En concreto, en aspectos como el acceso de las personas a la educación, a la salud, vivienda, la planificación sostenible y la gobernanza.

Este aumento de la complejidad de las áreas urbanas, ha motivado que desde mediados de los años 90 hayan aparecido nuevos enfoques de planificación y gestión urbana, que persiguen como norma general un desarrollo urbano más sostenible (Fernández, Pérez, Monzón y Torregrosa, 2015). En este contexto de cambio y ante la delicada situación económico-institucional, el enfoque de Ciudad Inteligente o Smart City ha tenido una gran acogida como modelo urbano de solución a la crisis, teniendo como argumentación básica la eficiencia (Fernández, 2015). La Unión Europea está impulsando la Smart City a través de un conjunto de iniciativas I+D+i en el marco de la Agenda Digital Europea. A nivel nacional, el impulso a las Ciudades Inteligentes ha venido dado a través de programas como el Plan Nacional de Ciudades Inteligentes (2015-2017), enmarcado en la Agenda Digital para España, y actualmente por el Plan Nacional de Territorios Inteligentes (2017-2020), que pretende dar continuidad al primer Plan Nacional de Ciudades Inteligentes (2015-2017).

Nos encontramos así con un nuevo paradigma de planificación y gestión urbana (Caragliu, Del Bo, y Nijkamp, 2011; Pierce, Ricciardi, y Zardini, 2017) que pretende ser la respuesta a los problemas de las ciudades del siglo XXI e implica la capacidad de las nuevas tecnologías para modernizar sus infraestructuras y servicios (Albino, Berardi, y Dangelico, 2015; Fernández-Güell, 2015). Es uno de los enfoques de planificación y gestión urbana más apreciados por los planificadores urbanos (Komninos y Tsarchopoulos, 2013), lo que explica que ya en 2012 la Comisión Europea hiciera suyo el modelo de ciudad inteligente basado en la conjunción de innovaciones tecnológicas en las áreas de energía, transporte y TIC (Fernández-Güell, 2015:21).

La ciudad inteligente presta especial interés en la aplicación de las nuevas tecnologías como pivote esencial para la obtención de una mejora en la calidad de vida, de las condiciones sociales, económicas y medioambientales de las áreas urbanas, gracias a su aplicación en la gestión de las infraestructuras (Guo, Liu, y Chai, 2014; IBM, 2010; Komninos, Schaffers, y Pallot, 2011; PwC y School, 2015). Las definiciones varían según el autor, ya que es un concepto en cierta medida emergente y por ello impreciso, todavía por clarificar (Albino, Berardi, y Dangelico, 2015; Caragliu, Del Bo, y Nijkamp, 2011; Nam y Pardo, 2011; Fernández, 2015; Fernández-Güell, 2015). Una de las definiciones más operativas es la de Giffinger (2007), que la define como "una ciudad que se desenvuelve con visión de futuro en la economía, las personas, la gobernanza, la movilidad, el medioambiente y la vida, basada en la combinación inteligente de dotaciones y actividades de ciudadanos autónomos, independientes y conscientes”. La ciudad inteligente se configuraría así como una centro de innovación que busca soluciones a los problemas económicos, sociales y ambientales de las urbes del siglo XXI (Vanolo, 2014). 
Para Caragliu, Del Bo y Nijkamp (2011), el concepto Smart City todavía está por clarificar, pero identifican características definitorias como:

- La utilización de una infraestructura de red para mejorar la eficiencia económica, y política, y posibilitar el desarrollo social, cultural y urbano.

- Un énfasis por la generación de espacios atractivos para la generación de negocios. Intentan conseguir la inclusión social en los servicios públicos ofertados.

- La apuesta por las industrias creativas y de alta tecnología.

- Una especial atención del capital social y relacional en el desarrollo urbano.

- La sostenibilidad social y medioambiental como aspecto estratégico de las Smart Cities.

Moreno (2015:30) sostiene que el concepto de Ciudad Inteligente gira ahora desde la dimensión meramente tecnológica hacia otras nuevas variables relacionadas con la calidad de vida, el desarrollo económico y la innovación, gracias a la integración tecnológica que permite conseguir unos servicios y procesos más eficientes. La tecnología y particular las TIC serían un medio para alcanzar la Ciudad Inteligente, de acuerdo a sus seis características principales: (1) e-Gobierno y e-Gobernanza, (2) movilidad, (3) sostenibilidad ambiental, (4) capital intelectual, (5) calidad de vida y (6) desarrollo económico.

A pesar de su interés y potencialidad, es un enfoque no exento de críticas (Datta, 2015; Fernández, 2015; Fernández-Güell, 2015; March y Ribera-Fumaz, 2014; Shelton, Zook, y Wiig, 2015; Kitchin, 2014), ya que a menudo suele identificarse como una etiqueta de moda alineada con agendas políticas e intereses empresariales, entre otros aspectos. Así, para Fernández-Güell (2015:21) las principales críticas provienen de un planteamiento excesivamente simplista y sesgado hacia la dimensión tecnológica, por lo que en los últimos años ha evolucionado hacia una visión holística de la Ciudad Inteligente como un sistema funcional complejo y multidimensional en el que los ciudadanos ganan importancia y comparten las decisiones con los agentes políticos y económicos. En esta misma línea, resultan especialmente interesantes las reflexiones de Fernández (2015: 44-45). Subraya el autor que la principal incógnita es conocer el papel que puede tener la Ciudad Inteligente en la ciudadanía, más allá de las promesas espectacularizadas basadas en escenarios futuristas, complejos diagramas de servicios urbanos interconectados y un lenguaje técnico muy alejado de la cotidianidad de la ciudadanía.

\subsection{La evolución tecnológica y su influencia en el turismo: Smart Tourism}

La reciente evolución tecnológica y la aparición de las Smart Cities como centros de innovación que buscan soluciones a los problemas económicos, sociales y ambientales de las urbes del siglo XXI (Vanolo, 2014), ha tenido su influencia en el sector turístico (Del Chiappa y Baggio, 2015). El concepto destino turístico inteligente es el resultado final de la traslación del enfoque urbano Smart City a los destinos turísticos (Baggio y Cooper, 2015) donde cobra especial relevancia al permitir evolucionar hacia 
una planificación sustentada en la generación de conocimiento y la innovación, con una fuerte componente tecnológica asociada (Huang, Goo, Nam, y Woo, 2017).

El concepto de ST en los países occidentales se acuña en el año 2000 de la mano de Gordon Philips (Li, Hu, Huang, y Duan, 2016) adoptando un enfoque holístico, a largo plazo y sostenible para planificar, desarrollar, operar y comercializar productos y negocios turísticos. En los países occidentales el ST no es una estrategia central del desarrollo turístico, sino que se ha fundamentado en su contribución al desarrollo sostenible y la relación entre turistas y destinos.

Como expone Gretzel, Sigala, Xiang y Koo (2015a), en Asia del Este, las acciones en torno al ST se centran en políticas que promueven el desarrollo de una infraestructura tecnológica, mientras que en Europa la mayoría de las iniciativas se identifican con proyectos de Smart City que han favorecido la aparición del enfoque destinos turísticos inteligentes. Aquí las iniciativas se asocian más a la innovación y competitividad, mediante desarrollo de aplicaciones para la mejora de la experiencia turística (SEGITUR, 2015). Por otro lado, en Australia, uno de los países más prolíficos en esta temática, el ST se enfoca hacia la mejora de los procesos de gobernanza a través de la apertura de datos.

Sin duda, este concepto puede considerarse también como un término novedoso que básicamente implica la aplicación de tecnologías inteligentes en todas las fases del viaje (Koo, Shin, Gretzel, Hunter, y Chung, 2016). El objetivo final es mejorar la experiencia turística y la competitividad del destino gracias a una gestión innovadora de la información que soporta el proceso de toma de decisiones. En los últimos años están surgiendo aproximaciones críticas respecto al uso intensivo de las TIC por parte del turismo, ya que no todo repercutiría en una mejora de la experiencia. Para Tribe y Mkono (2017), el ST no es tan inteligente, ya que las TIC pueden dar lugar a procesos de alienación, para lo que acuñan el término e-lienación.

Li, Hu, Huang, y Duan (2016) hacen una interesante aproximación al concepto smart. La definición inglesa de la palabra es wisdom (sabiduría), de acuerdo a la RAE el grado más alto del conocimiento; una conducta prudente en la vida o en los negocios; un conocimiento profundo en ciencias, letras o artes. Los autores hacen hincapié en diferenciar entre el concepto smartness y el concepto intelligence, aparentemente sinónimos (inteligencia) y utilizados en ocasiones de manera indistinta por los académicos. El concepto intelligence se centra en la capacidad técnica para ofrecer los servicios adecuados a los usuarios, también se relaciona con la experiencia o el resultado de una experiencia. Por otro lado, el concepto smartness enfatiza la facilidad con la que los usuarios pueden obtener automáticamente servicios adecuados. Según los autores, ser smart permitiría identificar las necesidades de los usuarios y ofrecerles los servicios de información adecuados gracias a la acumulación de información mediante dispositivos tecnológicos. Por tanto, el concepto smart no pone su centro de atención en la tecnología, sino en los resultados tecnológicos para las personas. Ser smart significaría identificar las necesidades de los usuarios para darles servicios adecuados a sus necesidades.

Autores como Hunter, Chung, Gretzel, y Koo (2015) entienden el ST como un fenómeno social que surge de la convergencia de las tecnologías de la información y 
la experiencia turística. La presencia ubicua y la influencia de Internet y los dispositivos móviles han generado nuevos modelos de negocio y nuevos tipos de experiencias turísticas, pero también nuevos problemas para los procesos de gestión de la imagen y marketing de los destinos. Para estos autores en el ST las personas construyen de manera conjunta ecosistemas sociales gracias al intercambio de información con dispositivos móviles conectados a internet, que construyen una realidad social del turismo o destino. Ahora la imagen de un destino se forma de manera ajena al destino, mediante el contenido que generan los usuarios en estos ecosistemas, como por ejemplo las redes sociales, a través de fotos, comentarios, etc. El ST se ha convertido en un aspecto esencial del turismo contemporáneo, haciendo la información más interactiva y atractiva para el turista, ofreciendo valores añadidos tanto para el turista como para el destino.

De acuerdo a Gretzel, Sigala, Xiang, y Koo (2015) el ST es una evolución lógica del turismo tradicional, y más recientemente, del denominado e-tourism, centrado en las conexiones business-consumers y consumer-consumers a través de la web. La clave para la innovación y la orientación tecnológica de la industria y los consumidores se estableció con la adopción de las tecnologías de la información y comunicación en el turismo, como por ejemplo los sistemas de distribución global y las centrales de reserva, o las tecnologías basadas en la web que permitieron el nacimiento del e-tourism. La evolución del turismo continuó con la adopción de las redes sociales y el nacimiento del turismo móvil, en íntima relación con la movilidad de la información turística y los consumidores turistas. Los autores consideran que el concepto ST supone una etapa distinta en la evolución de las TIC aplicadas al turismo, marcada por la conexión de lo físico con la gobernanza y el entorno digital, lo que genera nuevos niveles de inteligencia y cambios en las experiencias turísticas, en cómo se generan, como se consumen y se comparten. El concepto ST estaría formado por tres niveles diferentes (Smart Destinations, Smart Business Ecosystems y Smart Experience), cuyo aspecto clave es la integración de las TIC en infraestructura física, gracias al desarrollo del Internet of Things (IoT). Existiría un cuarto nivel, Smart Data, que se encargaría de recopilar datos (smart information), fomentar la interconectividad (smart exchange) $\mathrm{y}$, finalmente, analizar, visualizar, integrar y toma de decisiones (smart processing). A partir de estas consideraciones, definen el ST como "un turismo sustentado por los esfuerzos conjuntos del destino para obtener información de fuentes físicas y digitales que, combinadas con tecnologías avanzadas, son capaces de transformar los datos en experiencias e interesantes propuestas de valor enfocadas a la eficiencia, sostenibilidad y mejora de la experiencia turística".

Para el Smart Tourism Research Center (2017), uno de los grupos de investigación más activos en ST, las tecnologías de la información tienen un papel fundamental en los procesos de cambio, innovación y creación desarrollados en la industria turística desde sus orígenes contemporáneos hasta la actualidad. De acuerdo a este grupo, el turismo ha evolucionado básicamente en cuatro etapas, dominadas cada una de ellas por diferentes tecnologías que han desembocado en el ST. Lo definen como la prestación de servicios de información en tiempo real contextualizados para turistas, agentes, destinos e industrias, gracias a distintos sistemas de comunicación e información soportados por las TIC. 
Tabla 1

EVOLUCIÓN DEL TURISMO Y PRINCIPALES CAMBIOS TECNOLÓGICOS

\begin{tabular}{|c|c|c|c|}
\hline & \multicolumn{2}{|l|}{1950} & 2000 \\
\hline & $\begin{array}{c}\text { Turismo } \\
\text { tradicional }\end{array}$ & E-tourism & Smart Tourism \\
\hline $\begin{array}{l}\text { Tecnología } \\
\text { clave }\end{array}$ & Servidores & $\begin{array}{l}\text { Internet y la aparición } \\
\text { de la web. }\end{array}$ & $\begin{array}{l}\text { - Big Data } \\
\text { - Sensores } \\
\text { - Tecnologías móviles } \\
\text { - Internet de las Cosas } \\
\text { - Servicios Cloud }\end{array}$ \\
\hline $\begin{array}{l}\text { Elementos } \\
\text { clave del } \\
\text { sistema } \\
\text { turístico }\end{array}$ & $\begin{array}{l}\text { Sistemas } \\
\text { globales de } \\
\text { distribución } \\
(\mathrm{GDS}) \text { y las } \\
\text { centrales de } \\
\text { reserva (CRS). }\end{array}$ & $\begin{array}{l}\text { - Información a través } \\
\text { de la web. } \\
\text { - Existencia de } \\
\text { intermediarios. } \\
\text { - Intercambios B2B, } \\
\text { B2C y C2C }\end{array}$ & $\begin{array}{l}\text { - Personalización de } \\
\text { experiencias. } \\
\text { - Uso de tecnología } \\
\text { para procesos de co- } \\
\text { creación. } \\
\text { - Reintermediación } \\
\text { - Ecosistemas } \\
\text { colaborativos }\end{array}$ \\
\hline
\end{tabular}

Elaboración propia a partir de Buhalis y Amaranggana (2015), Gretzel (2011), Gretzel, Werthner, Koo, y Lamsfus (2015) y Zheng Xiang, Tussyadiah, y Buhalis (2015).

\section{METODOLOGÍA DE LA INVESTIGACIÓN}

Dada la alta componente tecnológica del concepto ST y el carácter multidisciplinar del turismo, se decide aplicar, por primera vez en el dominio turístico ${ }^{1}$, un estudio de mapeo sistemático (Systematic Mapping Study, SMS) de acuerdo a las orientaciones de Petersen, Feldt, Mujtaba, y Mattsson (2008). Esta técnica de estudio ha sido ampliamente utilizada desde hace años en disciplinas como la ingeniería informática (Kitchenham, Budgen, y Pearl Brereton, 2011) y junto con las revisiones sistemáticas de la literatura (Systematic Literature Review, SLR), son consideradas como estudios de carácter secundario (Kitchenham, 2010).

El mapeo sistemático es un método que se centra en la clasificación de la producción científica asociada a un determinado tema, para lo que categoriza y contabiliza los resultados o estudios primarios relacionados. La información resultante se combina para dar respuesta a un conjunto de preguntas de investigación, siguiendo para ello

1 Respecto a la técnica de estudio SMS, que adoptamos del dominio de la ingeniería informática, podemos afirmar que, como tal, es la primera vez que se aplica al ámbito turístico. Así queda justificado tras la consulta realizada en Scopus la primera semana de 2018. Se introduce la cadena de búsqueda "systematic mapping” AND "tourism". Se obtienen dos registros que, tras su análisis, se comprueba que no tienen ninguna relación con el dominio turístico. 
una estrategia o protocolo fundamentado en un proceso sistemático de revisión de la producción científica compuesto de una serie de etapas que se muestran en la Figura 1. Es una técnica de gran utilidad para temas de investigación muy amplios. El punto de partida del proceso de mapeo sistemáticos es la definición de los objetivos y preguntas de investigación, que guían la estrategia de búsqueda que permitirá identificar los trabajos más relevantes y que posteriormente serán clasificados para obtener el mapa visual del conocimiento existente. Como indican Kitchenham, Budgen y Brereton (2011), permite identificar temas donde existen un número suficiente de estudios primarios para desarrollar revisiones sistemáticas, además de tópicos que necesitan del desarrollo de más estudios primarios. Junto a las SLR, nos encontramos ante un tipo de estudio secundario.

\section{Figura 1 \\ ETAPAS DEL ESTUDIO DE MAPEO SISTEMÁTICO}

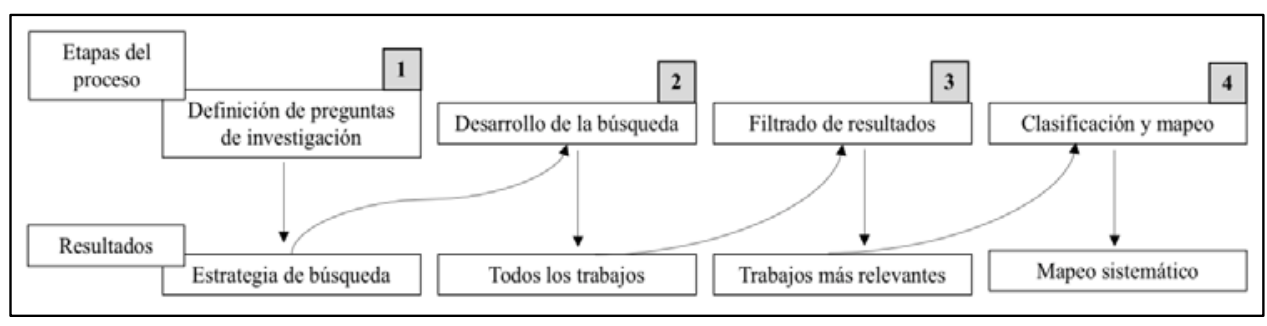

Elaboración propia a partir de Petersen, Vakkalanka, y Kuzniarz (2015).

Un SMS no es una SLR, a pesar de contar con similitudes, son métodos diferentes (Petersen, Vakkalanka, y Kuzniarz, 2015). Kitchenham, Budgen, y Pearl Brereton (2011) recogen algunas de las diferencias básicas de una SLR y un SMS. Encuentran cinco diferencias respecto al (1) tipo de preguntas de investigación, (2) proceso de búsqueda, (3) requisitos de la estrategia de búsqueda, (4) verificación de la calidad y (5) resultados. En este sentido, cabe destacar que la principal diferencia entre una SLR y un SMS es el tipo de pregunta a la que responde cada uno de ellos (1). Así, una SLR trata de responder a preguntas más específicas relativas a los resultados de los estudios, mientras que un SMS responde a una pregunta más general, relacionada con las tendencias de investigación en un tema determinado. Por otra parte, en un SMS el proceso de búsqueda (2) queda definido por un área de interés o topic, mientras que en una SLR el proceso de búsqueda se define por la pregunta de investigación. La estrategia de búsqueda (3) también es menos exigente en un SMS, ya que a diferencia de la SLR, no se buscan los estudios más relevantes, sino las tendencias de investigación en un tema de interés. Para un SMS la calidad de los estudios (4) no es esencial, pero una SLR debe contar con los trabajos con mejores resultados. Los resultados obtenidos (5) de un SMS son un conjunto de trabajos relacionados con una temática que son agrupados en categorías, mientras que en una SLR el resultado son respuestas a preguntas de investigación específicas. 
Tabla 2

DIFERENCIAS CLAVE ENTRE UN SMS Y UNA SLR

\begin{tabular}{|c|c|}
\hline Aspecto & Diferencias y similitudes \\
\hline $\begin{array}{l}\text { Preguntas de } \\
\text { investigación }\end{array}$ & $\begin{array}{l}\text { SLR: responde a preguntas específicas. Agrega resultados relacionados } \\
\text { con las preguntas de investigación. } \\
\text { SMS: responde a preguntas más abiertas, relacionadas con tendencias. } \\
\text { Se limita a identificar y clasificar estudios primarios en subtemas. } \\
\text { Por lo tanto, el análisis, la interpretación y la generalización de los } \\
\text { hallazgos está más focalizado. }\end{array}$ \\
\hline $\begin{array}{l}\text { Métodos de } \\
\text { búsqueda }\end{array}$ & $\begin{array}{l}\text { Ambos métodos se esfuerzan en encontrar los estudios relacionados } \\
\text { más relevantes, intentando así responder a las preguntas de } \\
\text { investigación, pero: } \\
\text { SLR: generalmente se centran en un tipo de evidencia. Ej. Artículos. } \\
\text { SMS: puede incluir diferentes tipos de estudios primarios. Ej. Tesis, } \\
\text { informes, etc. }\end{array}$ \\
\hline $\begin{array}{l}\text { Méto } \\
\text { selec }\end{array}$ & $\begin{array}{l}\text { Una de las diferencias más importantes entre ambos métodos la } \\
\text { encontramos en el proceso de selección de estudios. } \\
\text { SLR: suele seleccionar menos estudios, pero deben ser analizados en } \\
\text { mayor profundidad. } \\
\text { SMS: el alcance es más amplio. El análisis y síntesis más general. } \\
\text { Suele implicar más estudios. }\end{array}$ \\
\hline $\begin{array}{l}\text { Método de } \\
\text { extracción }\end{array}$ & $\begin{array}{l}\text { SLR: los datos a seleccionar de los estudios primarios pueden incluir } \\
\text { información detallada de los métodos y resultados de cada estudio, } \\
\text { una descripción de cada estudio y los resultados de cada estudio. } \\
\text { SMS: los datos a seleccionar de los estudios primarios pueden incluir } \\
\text { información bibliográfica de las publicaciones (Ej. Revista, fecha, } \\
\text { etc.) e información básica que describe la investigación que se ha } \\
\text { desarrollado (Ej. País, tipo de investigación, enfoque, etc.) }\end{array}$ \\
\hline Síntesis & $\begin{array}{l}\text { SLR: el principal objetivo es una síntesis complete de los resultados. } \\
\text { SMS: simplemente detalla información básica de cada estudio primario. } \\
\text { Ej. Método, procedencia geográfica, año de publicación, etc. Tabula los } \\
\text { estudios en categorías y lo sintetiza todo en un mapa o matriz. }\end{array}$ \\
\hline
\end{tabular}

Elaboración propia a partir de Napoleão, Felizardo, De Souza, y Vijaykumar, 2017.

\subsection{Trabajos relacionados}

La investigación turística ha ido aumentando de manera paralela al continuo crecimiento del turismo (Corral-Marfil y Cànoves, 2014), lo que ha conformado un campo de investigación maduro (Xiao y Smith, 2006) que justifica su revisión con la finalidad de avanzar en el conocimiento (Webster y Watson, 2002). Los análisis bibliométricos en turismo suponen una evaluación del conocimiento desarrollado en una determinada área de conocimiento y se han aplicado fundamentalmente a la valoración de revistas y autores 
que publican en las mismas (Hall, 2011). No obstante, y a diferencia de otras disciplinas, su aplicación en el turismo y la hospitalidad es muy reciente y data de 1989, cuando se publica el trabajo de Weaver y McCleary (1989). Como apuntan Albacete, Fuentes, y Haro-Domínguez (2013), éste fue el punto de partida de otros trabajos que se han centrado fundamentalmente en (1) la evaluación institucional, (2) análisis de autorías, (3) rankings de publicaciones, (4) evaluación del desarrollo de la disciplina a través del análisis del contenido de las publicaciones y (5) el análisis de las redes de investigadores en turismo.

Existen diferentes técnicas para investigar la producción científica y productividad en el ámbito turístico ( Benckendorff, 2009), no obstante la técnica más utilizada son las SLR (Koseoglu, Rahimi, Okumus, y Liu, 2016). Durante los últimos años se observa como el enfoque cuantitativo en las SLR está resultando ser más atractivo para determinados grupos (Pickering y Byrne, 2014; Pickering, Rossi, Hernando y Barros, 2018), lo que está derivando en investigaciones que combinan enfoques cualitativos y cuantitativos aplicados a la SLR (Yang, Khoo-Lattimore, y Arcodia, 2017).

Tal y como detalla Benckendorff (2009), se dispone de distintas técnicas para analizar las contribuciones científicas, temas y tendencias de un campo determinado, que van de las evaluaciones cualitativas a las cuantitativas. Las primeras pueden guardar cierto grado de subjetividad, mientras que en las cuantitativas encontramos la mayoría de las técnicas que forman parte de la bibliometría. A su vez, estas técnicas se pueden dividir en valorativas o relacionales. Las valorativas buscan evaluar el impacto del trabajo académico, para así comparar las contribuciones científicas de autores o instituciones. Las relacionales buscan relaciones dentro de la investigación, como la estructura de los campos de investigación, la aparición de nuevos temas y métodos, etc. De la combinación de técnicas surgen cuatro categorías.

- Cualitativas valorativas. Elaboración de rankings de revistas o autores de acuerdo a la opinión de expertos.

- Cuantitativas valorativas. Analizan la contribución de instituciones, revistas o autores a una determinada disciplina o campo.

- Cualitativas relacionales. Analizan temas clave, tendencias, metodologías y técnicas estadísticas.

- Cuantitativas relacionales. Incluyen el análisis de citas, de autores o artículos, de coocurrencias de términos, cocitas, etc.

Koseoglu, Rahimi, Okumus, y Liu (2016) realizan una completa aproximación a los estudios bibliométricos desarrollados en turismo desde el año 1988, para lo que analizan 190 artículos que incluyen análisis bibliométricos y que son recopilados de revistas de referencia en el ámbito del turismo. De su análisis concluyen que es fundamentalmente a partir del año 2008 cuando empiezan a publicarse este tipo de estudios y que, además, predominan las SLR (157). Los estudios bibliométricos valorativos (24) y relacionales (9) son escasos. Para los autores son tres los métodos bibliométricos que se pueden utilizar para analizar la producción científica de un campo o disciplina científica: (1) revisiones bibliográficas (SLR, meta-análisis y aproximaciones cualitativas), (2) técnicas valorativas (medidas de productividad, métricas de impacto y métricas híbridas) y (3) técnicas relacionales (análisis de citas, análisis bibliográfico, coocurrencias y co-auto- 
rías). Por otro lado, resulta muy clarificadora la clasificación que realizan de los estudios de revisión, de acuerdo al objeto fundamental de análisis. Distinguen entre estudios de revisión centrados en disciplinas; en temas de interés específicos; en metodologías, métodos o estadísticas; en casos; en autores, centros y países de referencia y revistas.

\subsection{Objetivos y preguntas de investigación}

El objetivo general de este estudio de mapeo sistemático es avanzar en el conocimiento de la producción científica asociada al concepto ST. De acuerdo a las orientaciones de Petersen, Feldt, Mujtaba y Mattsson (2008) y las conclusiones obtenidas del marco teórico planteado, se diseñan un total de nueve preguntas de investigación que permiten estructurar el conocimiento desarrollado hasta la fecha, justificando así el cumplimiento de los objetivos del estudio.

\section{Tabla 3}

\section{PREGUNTAS DE INVESTIGACIÓN}

\begin{tabular}{|l|l|}
\hline \multicolumn{1}{|c|}{ Pregunta } & \multicolumn{1}{c|}{ Objetivo } \\
\hline $\begin{array}{l}\text { PI-1. ¿Cuál es la evolución y } \\
\text { distribución de las investigaciones? }\end{array}$ & $\begin{array}{l}\text { Conocer la evolución de la investigación } \\
\text { entre en el período 2000-2017, así como su } \\
\text { distribución (artículos, capítulos de libro y } \\
\text { conferencias). }\end{array}$ \\
\hline $\begin{array}{l}\text { PI-2. ¿Cuál es la evolución y } \\
\text { distribución geográfica de las } \\
\text { publicaciones? }\end{array}$ & $\begin{array}{l}\text { Conocer la evolución de las investigaciones de } \\
\text { acuerdo de acuerdo a su procedencia geográfica. }\end{array}$ \\
\hline $\begin{array}{l}\text { PI-3. ¿Cuáles son los principales } \\
\text { tipos de investigación de acuerdo a } \\
\text { la procedencia geográfica? }\end{array}$ & $\begin{array}{l}\text { Identificar cuáles son los tipos de investigación } \\
\text { de acuerdo a la procedencia geográfica. }\end{array}$ \\
\hline $\begin{array}{l}\text { PI-4. ¿Cuáles son los principales } \\
\text { tipos de investigación } \\
\text { desarrollada? }\end{array}$ & $\begin{array}{l}\text { Conocer los tipos de investigación que se están } \\
\text { desarrollando en el estudio del ST. }\end{array}$ \\
\hline $\begin{array}{l}\text { PI-5. ¿Cuáles son los enfoques } \\
\text { utilizados? }\end{array}$ & $\begin{array}{l}\text { Identificar cuáles son los enfoques desarrollados } \\
\text { en cada una de las investigaciones. }\end{array}$ \\
\hline $\begin{array}{l}\text { PI-6. ¿Cuáles son los principales } \\
\text { temas de interés? }\end{array}$ & $\begin{array}{l}\text { Identificar los principales de temas de interés } \\
\text { abordados en los trabajos más relevantes. }\end{array}$ \\
\hline $\begin{array}{l}\text { PI-7. ¿Cuáles son los autores y los } \\
\text { trabajos más citados? }\end{array}$ & $\begin{array}{l}\text { Identificar los investigadores líderes en este } \\
\text { campo de investigación, así como los trabajos } \\
\text { más referenciados. }\end{array}$ \\
\hline $\begin{array}{l}\text { PI-8. ¿Cuáles son las tecnologías } \\
\text { asociadas al concepto ST? }\end{array}$ & $\begin{array}{l}\text { Identificar cuáles son las tecnologías asociadas } \\
\text { al ST. }\end{array}$ \\
\hline $\begin{array}{l}\text { PI-9. ¿Cuáles son las palabras } \\
\text { clave más utilizadas? }\end{array}$ & $\begin{array}{l}\text { Identificar las palabras clave más utilizadas en } \\
\text { los trabajos asociados más relevantes. }\end{array}$ \\
\hline
\end{tabular}




\subsection{Selección de base de datos y diseño de estrategia de búsqueda}

Para seleccionar los estudios primarios utilizamos la base de datos Scopus (Elsevier). Hasta el año 2004, Web of Science (WoS, Thomson Reuters) era la única herramienta de búsqueda bibliográfica disponible. A partir de esta fecha, Elsevier puso en marcha Scopus, actualmente la mayor base de datos multidisciplinar de citas y resúmenes de literatura revisada por pares: revistas científicas, libros y actas de congresos (Scopus, 2017). Contiene más de 60 millones de registros publicados en más de 21.500 revistas científicas (4.200 de acceso abierto), además de 360 publicaciones comerciales, 130.000 libros y 1.100 series de libros, entre otras características. La mayoría de estos registros incluyen referencias que datan de 1996.

La elección de esta base de datos queda justificada de acuerdo a su mayor cobertura en revistas científicas relacionadas con el turismo (Benckendorff y Zehrer, 2013;Hall, 2011; McKercher, 2008) y, por otra parte, el período de búsqueda seleccionado: 2000-2017. En este sentido, la cobertura de WoS y Scopus a partir de 1996 es muy similar (Goodman y Deis, 2005;2007), por lo que son herramientas complementarias de acuerdo a la estrategia de búsqueda. Del mismo modo, se observa un alto grado de correlación en el número de trabajos y citas existentes en WoS y Scopus (Archambault, Campbell, Gingras, y Larivière, 2009), por lo que también se considera la herramienta más apropiada para esta investigación. Sus ventajas y desventajas han sido analizadas por diferentes autores (Bar-Ilan, 2010; Chadegani, Salehi, Yunus, Farhadi, Fooladi y Farhadi, 2013; Falagas, Pitsouni, Malietzis, y Pappas, 2007; Goodman y Deis, 2007; Vieira y Gomes, 2009).

A continuación, con el fin de realizar la búsqueda de la producción científica asociada al concepto ST, se definen las etiquetas o cadenas de búsqueda a consultar. Inicialmente y con el propósito de conocer la amplitud de los trabajos potencialmente relevantes, se opta por utilizar la etiqueta Smart Tourism (sin comillas) y aplicar la búsqueda al título, resumen y palabras clave.

\subsection{Filtrado de resultados}

Después de obtener estos primeros resultados, se procede a su filtrado de acuerdo a los criterios de inclusión y exclusión definidos. Se incluyen los trabajos que cumplen CI1, CI2 y CI3, mientras que se excluyen aquellos que cumplan con CE1 o CE2 o CE3 o CE4 o CE5.

\section{(1) Criterios de inclusión}

CI1: Todos los artículos, capítulos de libros y conferencias publicados en inglés que sigan un proceso de peer-review y tengan sus textos completos disponibles.

CI2: Las investigaciones deben incluir en el título, resumen o palabras clave el término ("smart tourism") O ("smart city" y "tourism") O el término ("smart destination") O ("smart tourism destination") O sinómimos o variables de los términos. Por ejemplo, "tourist" en lugar de "tourism", "cities" en lugar de "city", "destinations" en lugar de "destination".

CI3: El turismo debe ser el centro de atención de las investigaciones, no un tema tangencial. 


\section{(2) Criterios de exclusión}

CE1. Todos los artículos, capítulos de libros y conferencias publicados con anterioridad al año 2000.

CE2. Trabajos que no han pasado un proceso de peer-review (prefacios, editoriales, etc.).

CE3. Trabajos de los que no se dispone de sus textos completos.

CE4. Trabajos en idiomas diferentes al inglés

CE5. Las investigaciones no incluyen en el título, resumen o palabras clave el término ("smart tourism") O ("smart city" y "tourism") O el término ("smart destination") $\mathrm{O}$ ("smart tourism destination") O sinómimos o variables de los términos. Por ejemplo, "tourist" en lugar de "tourism", "cities" en lugar de "city", "destinations" en lugar de "destination".

\subsection{Clasificación de los trabajos}

Tras el proceso de filtrado se obtiene el conjunto de trabajos más relevantes, que son clasificados para responder a las preguntas de investigación establecidas. Tras la lectura del título, resumen y palabras clave, los trabajos se clasifican de acuerdo a tres categorías: enfoque, el tipo de aportación y la temática de la investigación en el que se encuadran. En los casos en los que no es suficiente con esta información para la clasificación del trabajo, se recurre al texto completo. Los procesos indicados en el punto 3.3 y 3.4, son realizados por dos autores de manera individual, que posteriormente ponen en común sus resultados para comprobar posibles discrepancias. En caso de existir, se recurre a un tercero para dirimir los resultados finales.

\subsubsection{Enfoque de la investigación}

De acuerdo a Gretzel, Sigala, Xiang y Koo (2015), establecemos tres enfoques fundamentales de las investigaciones sobre ST: Smart Destinations, Smart Experience y Smart Business. Añadimos un cuarto, Smart Tourism, ya que hay trabajos que no encajan en ninguno de los tres enfoques anteriores, por ejemplo, las reflexiones teóricas.

- Smart Destinations. Trabajos que analizan los principios de la ciudad inteligente o Smart City aplicados a destinos turísticos o Smart Destinations. Un aspecto clave de estos destinos es la conexión del mundo físico y digital, mediante tecnologías como el Internet de las Cosas (IoT). También se clasifican en este enfoque trabajos que aplican claramente tecnología a un destino.

- Smart Experience. Trabajos que analizan la tecnología y su relación con la mejora de la experiencia turística gracias a la personalización y la participación de los turistas en la creación de sus experiencias, gracias a la información que también proporcionan mediante comentarios y datos on-line generados.

- Smart Business. Trabajos que abordan la tecnología desde una perspectiva relacionada con la empresa, con las instituciones públicas y la creación de políticas relacionadas con la gobernanza, creación de infraestructuras, programas de desarrollo, etc. 
- Smart Tourism. Trabajos que no se pueden clasificar fácilmente en ninguna de las categorías anteriores. Se incluyen aquí trabajos que se centran en el análisis del concepto desde diferentes perspectivas y/o abarcan distintos enfoques, por lo que no hacen posible su inclusión en los enfoques anteriores.

\subsubsection{Tipo de investigación}

De acuerdo al tipo de investigación desarrollada, se establece la clasificación que se detalla a continuación.

- Teórica o Conceptual (T-C). Trabajos que desarrollan la vertiente teórica o conceptual relacionada con el concepto ST aportando básicamente un estado de la cuestión, propuestas y/o modelos teóricos.

- Estudios de casos (E-C). Trabajos orientados hacia el análisis de casos de estudio específicos (regionales, estatales o locales) donde se aplica el concepto ST.

- Reflexiones críticas (R-C). Trabajos cuyo objeto fundamental es aportar una valoración crítica en torno al concepto ST.

- Aportaciones metodológicas (A-M). Trabajos que tienen como aportación esencial una metodología específica que aporta valor al concepto ST, en todas sus variables.

- Aplicación tecnológica (A-T). Trabajos que proponen la aplicación tecnológica como aportación fundamental.

\subsubsection{Temática de la investigación}

Las temáticas de las investigaciones son definidas de acuerdo a los topics propuestos por el congreso ENTER2018, organizado por la Federación Internacional de Tecnología de la Información y Viajes y Turismo (IFITT). Este congreso surge en Innsbruck (Austria) en el año 1994 y se configura como un hito en la investigación del turismo y la tecnología (Buhalis y Law, 2008). Dada la alta componente tecnológica del concepto ST, adoptamos las temáticas de investigación de ENTER2018. Añadimos una cuarta categoría, "Otro", para clasificar los trabajos que no encajan en ninguno de las temáticas anteriores.

- Gobernanza y modelos de negocio (GOV). Son investigaciones claramente orientadas a empresas, corporaciones o instituciones. Centran su interés en temas relacionados con estrategias digitales en empresas y/o corporaciones (E-strategy, e-Business model); plataformas digitales; estrategias y sistemas avanzados de distribución; paquetes dinámicos; trabajos que abordan las TIC como posibilitadoras de los procesos de colaboración y asociación; trabajos que abordan las TIC para la innovación y el diseño de productos; educación a distancia, educación permanente y MOOCs; trabajos que abordan las nuevas tecnologías para el desarrollo regional y la sostenibilidad; trabajos que abordan aspectos legales y sociales relacionadas con las TIC; trabajos relacionados con el gobierno electrónico y políticas públicas en turismo; trabajos que abordan la brecha digital y el desarrollo socioeconómico. 
- Sistemas de información, plataformas tecnológicas y ciencia de los datos (SIS). Trabajos que abordan tecnologías relacionadas con la ciencia de los datos (Big Data, minería de textos, trabajos analíticos relacionados con la medición de variables); tecnologías móviles y tecnologías portables (wearable technologies); servicios basados en la ubicación y el contexto; Internet de las Cosas (IoT) y Destinos Inteligentes; búsqueda y obtención de información para viajes; trabajos que abordan el "Yo cuantificado" (Quantified Self); Web geoespacial: Inteligencia artificial, el aprendizaje de máquinas (Machine learning) y robots; la tecnología Blockchain; trabajos de la web semántica; trabajos relacionados con la privacidad y seguridad de los datos.

- Marketing Digital (MD). Trabajos relacionados con estrategias de marketing basadas en el juego (gaming); tecnologías como la realidad aumentada y/o realidad virtual; diseño y evaluación de web; estrategias de social media y marketing electrónico; trabajos relacionados con redes sociales, Social Media e inspiración social; trabajos que abordan las TIC y su influencia en la experiencia turística; trabajos que abordan la adopción, uso y el valor de las nuevas tecnologías.

- Otro (O). Cualquier otro tema de interés no considerado en las categorías anteriores.

\section{ANÁLISIS Y DISCUSIÓN DE RESULTADOS}

La búsqueda inicial con la etiqueta Smart Tourism (sin comillas) fue realizada durante la primera semana de 2018, devolviendo 540 resultados. Tras la aplicación de los criterios de inclusión y exclusión se obtienen un total de 162 trabajos, que pasan a ser analizados y clasificados de acuerdo a las conclusiones alcanzadas tras la lectura del título, resumen y palabras clave de cada una de ellas. En este proceso intervienen dos investigadores que realizan una primera clasificación de manera independiente. Después, existe una reunión para poner en común los resultados y resolver conflictos. Finalmente, un tercer investigador más experimentado resuelve los conflictos que no hayan podido ser resueltos. A continuación, se procede a resolver las preguntas de investigación que han guiado este estudio de mapeo sistemático.

\section{Figura 2 \\ ESQUEMA DEL PROCESO DE BÚSQUEDA}

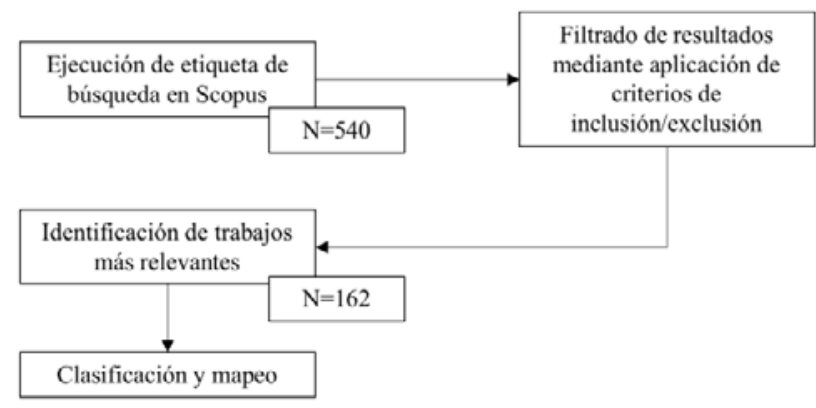

Elaboración propia. 


\subsection{Evolución de las publicaciones, procedencia geográfica y tipo de investigación desarrollada (PI-1, PI-2, PI-4)}

El proceso de búsqueda se ha centrado en el período 2000-2017. Se inicia en el año 2000 porque es en este año cuando se define el concepto ST, de acuerdo a Li, Hu, Huang, y Duan (2016). A pesar de haber sido acuñado en el año 2000, no es hasta el 2011 cuando se identifica el primer trabajo científico. Esto parece corroborar que es un concepto surgido del ámbito empresarial. Es fundamentalmente a partir del año 2014 cuando la producción científica en torno al concepto ST empieza a desarrollarse de una manera clara, con 12 trabajos. En el año 2016 se publican un total de 35 estudios, cifra que se duplica en el año 2017 con 75 publicaciones (Gráfico 1).

\section{Gráfico 1 \\ EVOLUCIÓN TEMPORAL DE LAS PUBLICACIONES}

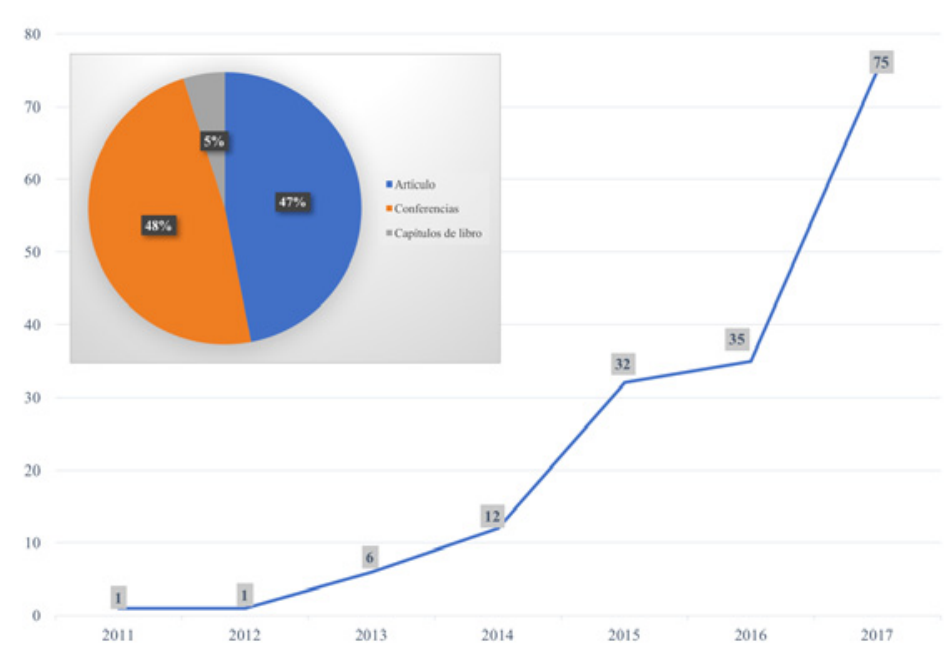

Elaboración propia.

Respecto a la procedencia de los trabajos (Gráfico 2), indicar que se establece de acuerdo al país de adscripción del primer autor. Cinco países producen el 64,8\% de las publicaciones seleccionadas: China $(22,8 \%)$, Italia $(19,1 \%)$, Corea del Sur $(13,6 \%)$, España $(6,2 \%)$ y Estados Unidos $(3,1 \%)$. En China destacan las aportaciones metodológicas $(35,1 \%)$, los estudios de casos (27\%) y las aplicaciones tecnológicas (27\%). El 65\% de los trabajos se concentran en el año 2017. Italia aporta investigaciones centradas en aplicaciones tecnológicas $(32,2 \%)$, aportaciones metodológicas (29\%) y estudios de casos $(25 \%)$. El $51,6 \%$ de los estudios italianos se publican entre 2016 y 2017. Por otro lado, la mitad de las publicaciones españolas son aplicaciones tecnológicas y un 30\% estudios de casos. Los trabajos españoles surgen a partir del año 2015 con cuatro publicaciones. En 2016 tan solo se encuentra un trabajo. En 2017 se identifican 5 publicaciones. Los trabajos estadounidenses son mayormente aplicaciones tecnológicas (40\%) y aportaciones metodológicas (40\%). 


\section{Gráfico 2 \\ MATRIZ DE DOS DIMENSIONES: PAÍSES, AÑOS Y TIPO DE INVESTIGACIÓN}

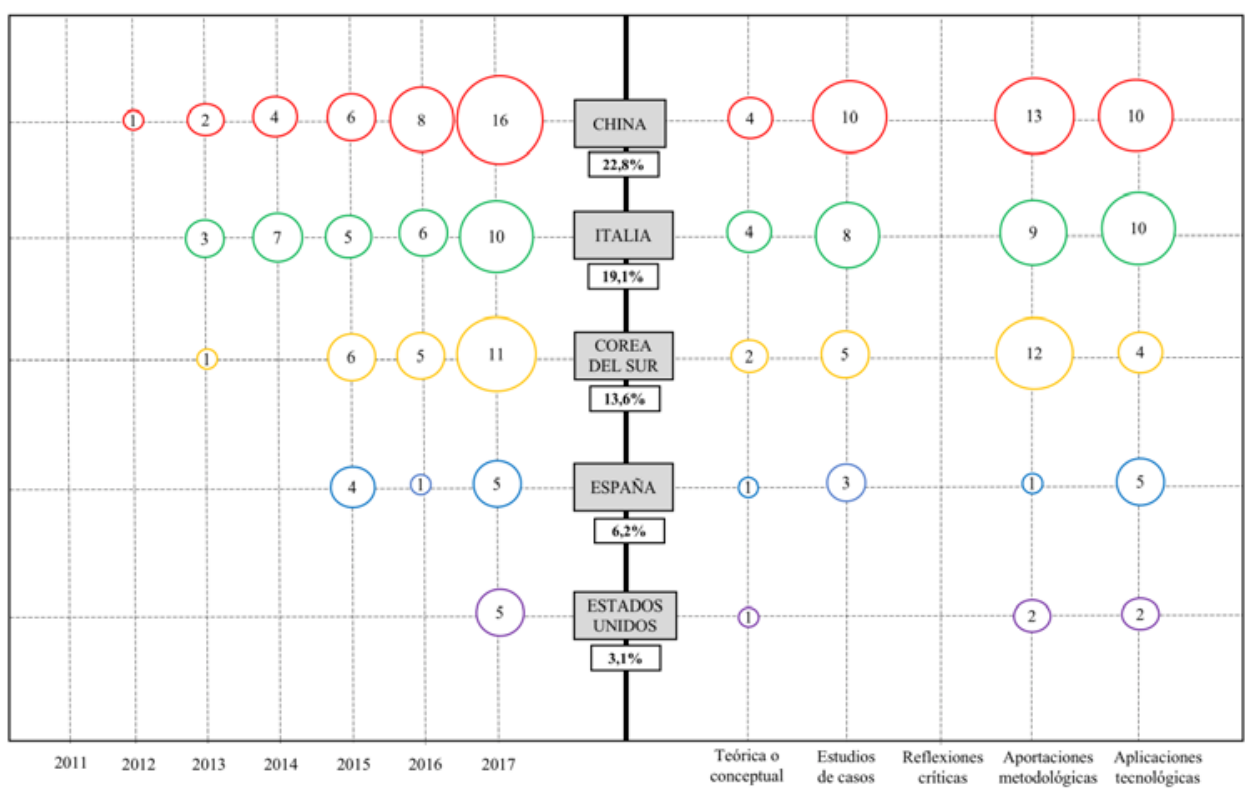

\subsection{Tipo de investigación desarrollada, principales enfoques y temas de interés (PI-4, PI-5, PI-6)}

Entre los tipos de investigación más utilizados (Gráfico 3), destacan las aplicaciones tecnológicas (32\%), las aportaciones metodológicas (31\%) y los estudios de casos (25\%). Las trabajos teórico-conceptuales son escasos, tan solo un $11 \%$ de los estudios abordan esta orientación. Las reflexiones críticas son prácticamente inexistentes (1\%). Los estudios de aplicación de tecnología y aportaciones metodológicas presentan una evolución muy favorable en 2017.

Respecto a la temática (Gráfico 4), destacan los trabajos que abordan los sistemas de información, plataformas tecnológicas y ciencia de los datos (42\%), seguidos del enfoque de marketing digital (30\%) y la gobernanza y modelos de negocio (21\%). En el 2017, los temas que mayor crecimiento han presentado respecto al año anterior han sido los sistemas de información, plataformas tecnológicas y ciencia de los datos (30 trabajos), junto al marketing digital (23 trabajos).

En cuanto al enfoque de la investigación (Gráfica 5), predominan las englobadas en la categoría Smart Experience (36\%), seguidas del enfoque Smart Destinations (29\%), Smart Tourism (19\%) y Smart Business (16\%). El enfoque que más está creciendo es el de Smart Experience y el de Smart Destination. 


\section{Gráfico 3}

EVOLUCIÓN Y TIPO DE INVESTIGACIÓN

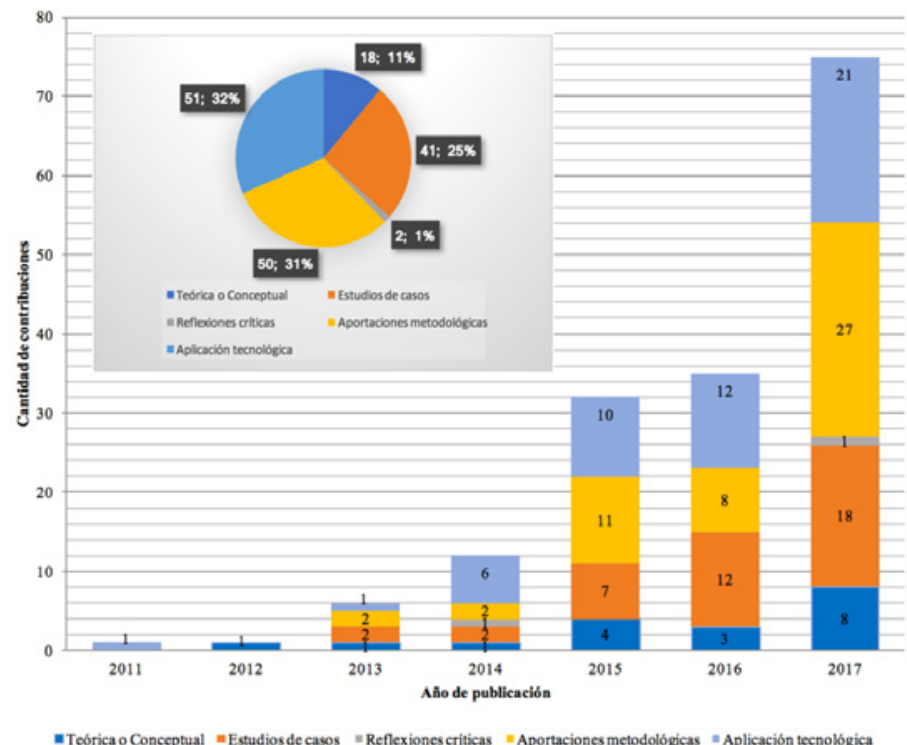

Elaboración propia.

\section{Gráfico 4 \\ EVOLUCIÓN Y TEMA DE INTERÉS}

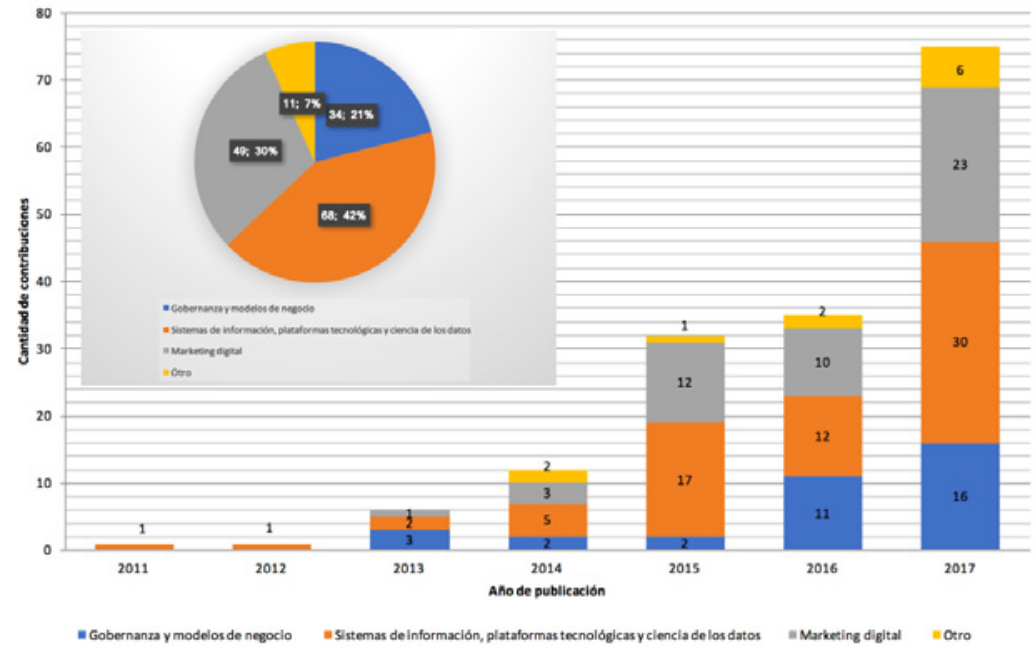

Elaboración propia. 


\section{Gráfico 5 \\ EVOLUCIÓN Y ENFOQUE DE LA INVESTIGACIÓN}

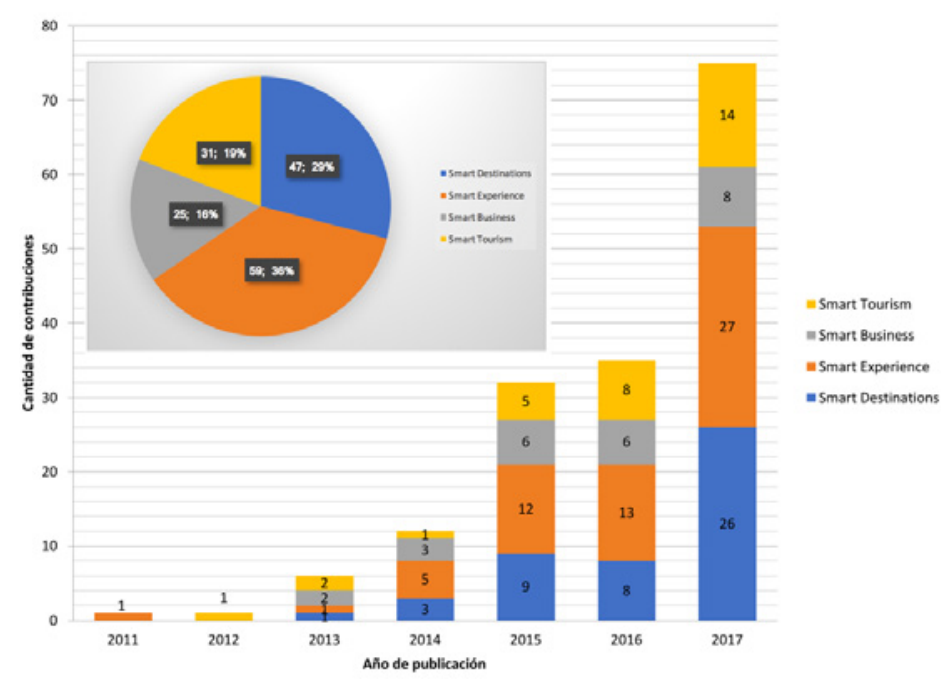

Elaboración propia.

El Gráfico 6 es una matriz bidimensional que tiene en su eje vertical el tipo de investigación, que correlaciona con el tema de interés (izquierda) y enfoque adoptado (derecha). El análisis de esta matriz aporta reflexiones muy interesantes. La aplicación de tecnología ha sido el tipo de investigación más desarrollado: un 32\% de los trabajos identificados resultaron seguir esta tipología. Del mismo modo, dentro de la misma, ha predominado un enfoque Smart Experience (26 trabajos) orientado a la mejora de la experiencia turística a través de las nuevas tecnologías, seguido del enfoque Smart Destinations (12 trabajos), identificado a su vez con estudios que aplican la tecnología a destinos. Respecto al tema de interés desarrollado en estos trabajos de aplicación de tecnología, se han centrado en los sistemas de información, plataformas tecnológicas y ciencia de los datos (31). En esta temática, los trabajos relacionados con Big Data y la ciencia de los datos son numerosos. La temática Marketing digital (16) es la segunda más importante, con trabajos relacionados con las TIC y la mejora de la experiencia. Los trabajos de aplicación tecnológica orientados a la gobernanza y modelos de negocios son minoritarios (4). El siguiente tipo de investigación más desarrollado son las aportaciones metodológicas (31\%), que se centran fundamentalmente en el enfoque Smart Experience (21) y Smart Destinations (13), mientras que su temática predilecta es el Marketing digital (20) y los sistemas de información, plataformas tecnológicas y ciencia de los datos (17). Resulta llamativo que las reflexiones críticas (1\%) no tienen un especial atractivo para los investigadores, ya que tan solo cuenta con los trabajos de Tribe y Mkono (2017) y Graziano (2014). Es por tanto una línea de investigación muy poco desarrollada. Los estudios de casos (25\%) son el tercer tipo de investigación más desarrollado. Su enfoque principal es el de Smart Destinations (17), mientras que se 
centran en la temática de Sistemas de infomación, plataformas tecnológicas y ciencia de los datos (15) y Gobernanza y modelos de negocio (13). Por último, los estudios de carácter teórico-conceptual $\mathbf{( 1 1 \% )}$ ) no tienen un enfoque prioritario, aunque el enfoque Smart Destinations (5) está a la cabeza. Por otro lado, la temática Gobernanza y modelos de negocio es la más utilizada en los estudios teóricos (6), aunque tampoco existe una claro dominancia.

\section{Gráfico 6}

MATRIZ DE DOS DIMENSIONES: TIPO DE INVESTIGACIÓN, TEMA DE INTERÉS Y ENFOQUE

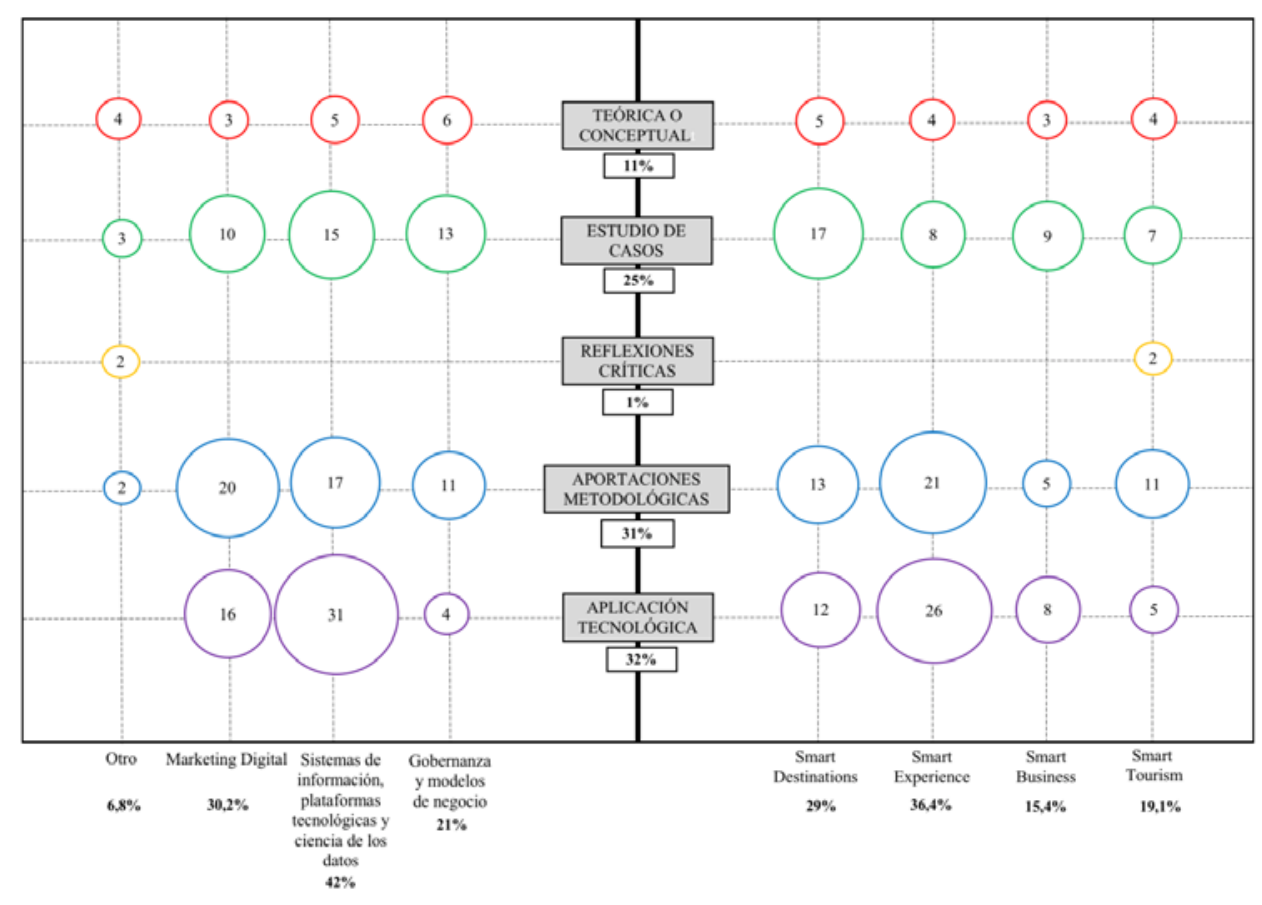

\subsection{Autores y trabajos más citados, tecnologías asociadas y palabras clave más uti- lizadas (PI-7, PI-8, PI-9)}

Con el objeto de mostrar una primera aproximación a los autores más productivos en el ámbito del ST, desde una perspectiva cuantitativa, no relacional y sintética, la Tabla 4 muestra los 5 autores más productivos de acuerdo al número citas obtenidas en Scopus. La región de Asia del Este lidera la lista de los autores más productivos. Destaca en este sentido la producción académica de Chulmo Koo, director gerente del Smart Tourism Research Center (STRC, Corea del Sur), con 191 citas y un total de 8 trabajos relacionados. La segunda posición la ocupa la investigadora de adscripción 
estadounidense Ulrike Gretzel, con 2 trabajos relacionados y una ratio de 56, más del doble de la de Chulmo Koo, dado su menor número de trabajos. El también surcoreano Namhoo Chung, director de STRC, ocupa la tercera posición con 102 citas y un total de 11 trabajos, pero la tercera ratio más baja del conjunto $(9,3)$. Destacan por su ratio Zheng Xiang y Marianna Sigala, con una publicación relacionada y 74 citas. No obstante, estas 74 citas provienen del trabajo conjunto de Gretzel, Sigala, Xiang y Koo (2015): "Smart Tourism: foundations and developments".

\section{Tabla 4 \\ AUTORES MÁS PRODUCTIVOS RESPECTO AL NÚMERO TOTAL DE CITAS}

\begin{tabular}{|c|c|c|c|c|}
\hline Autor & País de adscripción & $\begin{array}{c}\mathbf{N}^{\mathbf{o}} \text { Trabajos } \\
\text { relacionados }\end{array}$ & Citas & Ratio citas \\
\hline Chulmo Koo & Corea del Sur & 8 & 191 & 23,9 \\
\hline Ulrike Gretzel & EEUU & 2 & 112 & 56,0 \\
\hline Namho Chung & Corea del Sur & 11 & 102 & 9,3 \\
\hline Zheng Xiang & EEUU & 1 & 74 & 74,0 \\
\hline Marianna Sigala & Australia & 1 & 74 & 74,0 \\
\hline
\end{tabular}

Elaboración propia.

Como se recoge en la Tabla 5, la publicación más citada es la de Gretzel, Sigala, Xiang y Koo (2015), con 74 citas. En segundo lugar, se encuentra el trabajo de los coreanos Chung y Koo (2015), con 50 citas. En tercer y cuarto lugar, encontramos las publicaciones de Sun, Song, Jara y Bie (2016) y Wang, Li, y Li (2013), con 46 y 40 citas, respectivamente. El trabajo de Gretzel, Werthner, Koo y Lamsfus (2015) ocupa el quinto lugar con 38 citas.

Para identificar las tecnologías asociadas al concepto ST, se analizan las palabras clave de los trabajos de acuerdo a las tecnologías identificadas por Ivars-Baidal, Celdrán-Bernabeu, Mazon y Perles-Ivars (2017). De este modo, la tecnología que más se asocia es el Internet de las Cosas (IoT), con un $39 \%$ respecto al total de tecnologías que se identifican. Mediante el uso de distintos sensores, la tecnología IoT permite conectar el mundo físico con el digital. Por otro lado, encontramos la tecnología Big Data (29\%) que es aplicada a grandes volúmenes de datos para la obtención de conocimiento, con diferentes finalidades. La Realidad Aumentada (9\%), permite superponer información digital sobre una imagen de algo que está siendo observado mediante un dispositivo móvil, lo que puede favorecer una mejora de la experiencia del turista. La tecnología NFC (Near Field Communication) es un sistema de comunicación inalámbrico integrado en smartphones y tablets que permite mejorar la experiencia del turista. Del mismo modo, la tecnología de comunicación óptica Li-Fi está ganando cada vez más atención. Estas dos últimas tecnologías ocupan el quinto lugar, con un $5 \%$. 


\section{Tabla 5 \\ TRABAJOS MÁS CITADOS}

\begin{tabular}{|c|c|c|c|c|c|}
\hline Autores & Título & Año & Medio & Citas & Clasificación \\
\hline $\begin{array}{c}\text { Gretzel U., } \\
\text { Sigala M., } \\
\text { Xiang Z., Koo } \\
\text { C. }\end{array}$ & $\begin{array}{l}\text { Smart tourism: } \\
\text { foundations and } \\
\text { developments }\end{array}$ & 2015 & Electronic Markets & 74 & $\mathrm{~T}-\mathrm{C} / \mathrm{SIS} / \mathrm{ST}$ \\
\hline $\begin{array}{c}\text { Chung N., Koo } \\
\text { C. }\end{array}$ & $\begin{array}{l}\text { The use of social } \\
\text { media in travel } \\
\text { information search }\end{array}$ & 2015 & $\begin{array}{l}\text { Telematics and } \\
\text { Informatics }\end{array}$ & 50 & A-M/SIS/SE \\
\hline $\begin{array}{l}\text { Sun Y., Song } \\
\text { H., Jara A.J., } \\
\text { Bie R. }\end{array}$ & $\begin{array}{l}\text { Internet of Things and } \\
\text { Big Data analytics for } \\
\text { smart and connected } \\
\text { communities }\end{array}$ & 2016 & IEEE Access & 46 & A-T/SIS/ST \\
\hline $\begin{array}{l}\text { Wang D., Li X., } \\
\text { Li Y. }\end{array}$ & $\begin{array}{c}\text { China's smart tourism } \\
\text { destination initiative: } \\
\text { a taste of the service } \\
\text { dominant logic } \\
\end{array}$ & 2013 & $\begin{array}{c}\text { Journal of } \\
\text { Destination } \\
\text { Marketing and } \\
\text { Management }\end{array}$ & 40 & E-C/GOV/SD \\
\hline $\begin{array}{l}\text { Gretzel U., } \\
\text { Werthner } \\
\text { H., Koo C., } \\
\text { Lamsfus C. }\end{array}$ & $\begin{array}{c}\text { Conceptual } \\
\text { foundations for } \\
\text { understanding smart } \\
\text { tourism ecosystems }\end{array}$ & 2015 & $\begin{array}{c}\text { Computers in } \\
\text { Human Behavior }\end{array}$ & 38 & $\mathrm{~T}-\mathrm{C} / \mathrm{GOV} / \mathrm{SB}$ \\
\hline
\end{tabular}

Elaboración propia.

En la nube de palabras representada por la Figura 3 podemos observar las palabras clave más utilizadas en los trabajos seleccionados. Destaca a este respecto la existencia de numerosos términos tecnológicos que vienen a corroborar la importante carga tecnológica asociada al ST: mobile, IoT, user, gis, internet, network, ecosystems, semantics, telecom, api, web. Resaltar además que excluyendo el término más importante ("tourism"), los dos términos que le siguen en número de apariciones son "mobile" (destacando la importancia de los dispositivos móviles en este campo) e "information" (igualmente importante por la relevancia de la información que se genera y gestiona asociada al término "Smart Tourism”). Por otra parte es relevante también la aparición de dos términos crecientes en su grado de importancia como "smart city" y "IoT". Destacar la ausencia del término "data" que queda relegado por el de "information". También destacar la palabra "integrated" que da una idea de la necesidad de integración de tecnologías y otros conceptos que es necesario para "Smart Tourism". Se podrían clasificar los términos en:

- De dominio: tourism, travel, location, air transportation, planning, marketing, urban, services.

- Tecnológicos: mobile, information, smart city, telecom, semantics, internet, network, IoT, GIS, web, API

- Transversales: sustainability, ecosystem, integrated, user, research, 


\section{Gráfico 6}

\section{TECNOLOGÍAS ASOCIADAS AL CONCEPTO ST}

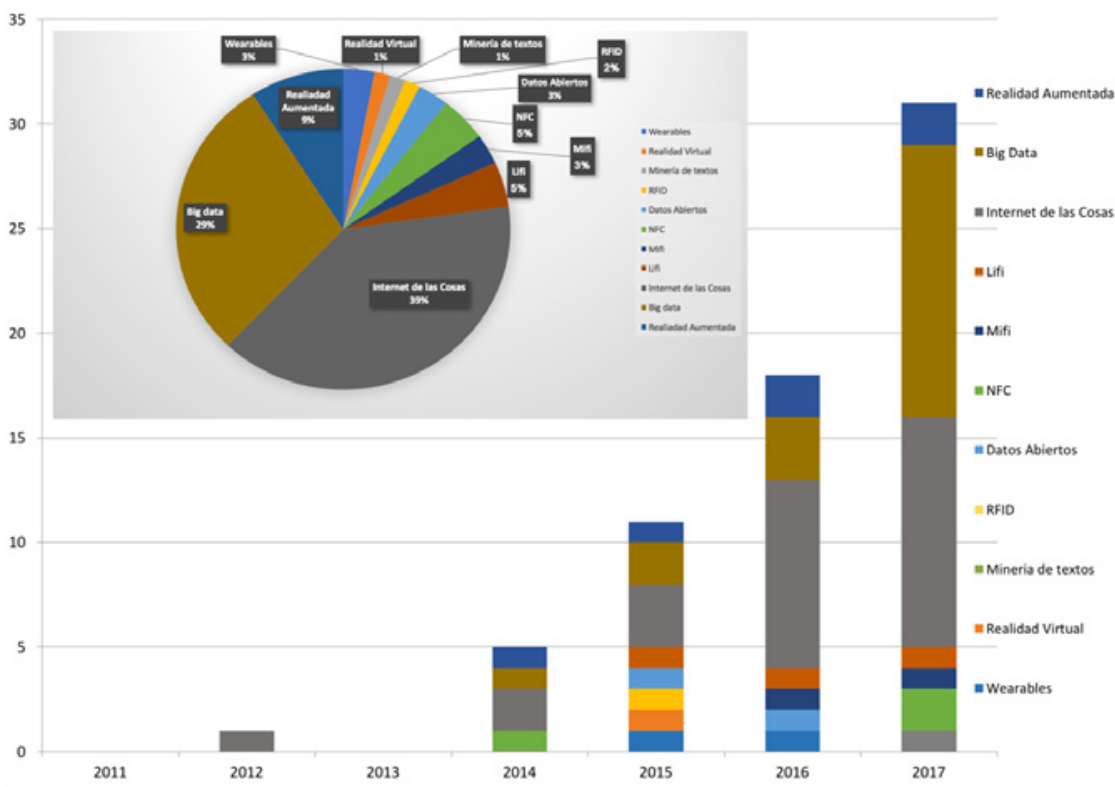

Elaboración propia.

Figura 3

PALABRAS CLAVE MÁS UTILIZADAS

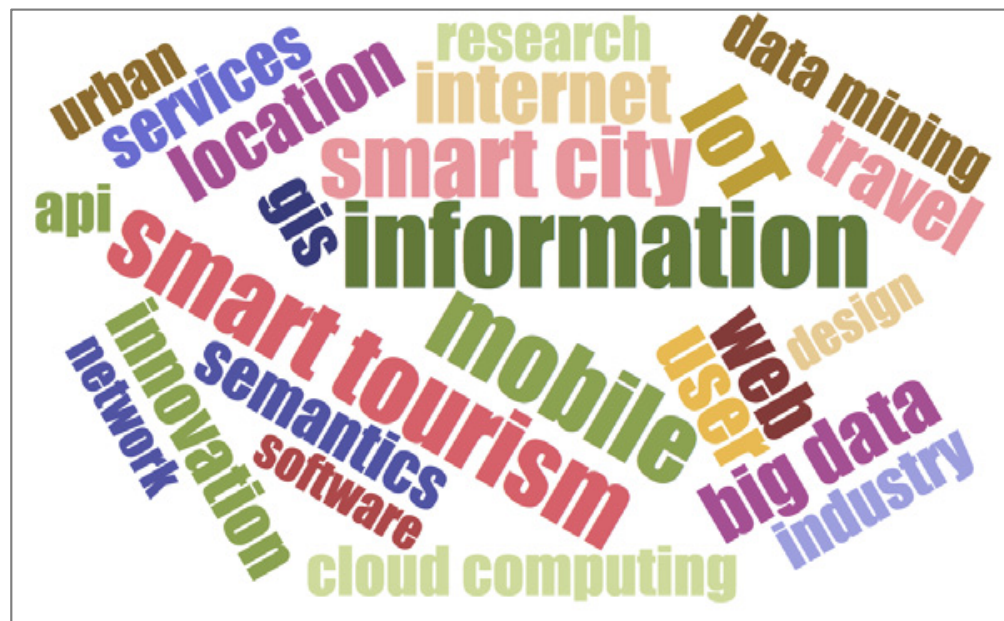

Elaboración propia. 


\section{CONCLUSIONES}

En este trabajo se ha desarrollado un estudio de mapeo sistemático con el objetivo de caracterizar la producción científica relacionada con el concepto Smart Tourism. Esta técnica, propia de la ingeniería del software, ha sido aplicada por primera vez al dominio turístico y, en concreto, a un concepto novedoso, muy amplio, y con una componente tecnológica importante. El proceso de análisis y clasificación de los estudios primarios se inicia con el diseño de la estrategia de búsqueda definida por un total de 9 preguntas de investigación. Como resultado, se seleccionan un total de 162 trabajos que son clasificados de acuerdo al tipo de investigación, el enfoque utilizado y la temática abordada. Utilizando información complementaria, como la relativa a la procedencia geográfica, autores más productivos, trabajos más citados, tecnologías asociadas y palabras clave más utilizadas, se consigue dar respuesta a las preguntas de investigación iniciales y avanzar en el conocimiento de este concepto.

A pesar de que autores como Li, Hu, Huang y Duan (2016) indican que este concepto se acuña el año 2000 por Gordon Phillips, la realidad es que la producción científica en torno al ST no empieza a evolucionar de manera clara hasta el año 2013, cuando se publican 6 trabajos. En el año 2014 se publican 12 trabajos, para pasar a 32, en 2015, y 35, en 2016. En 2017 parece existir un punto de inflexión, alcanzándose la cifra de 75 publicaciones. Es por lo que podemos afirmar que la investigación existente en este campo se encuentra en un estado preliminar o emergente, con muy pocos trabajos teórico-conceptuales $(11 \%)$ que recojan los aspectos esenciales del ST. Del mismo modo, las reflexiones críticas (1\%) son prácticamente inexistentes, aunque planteamientos críticos están recogidos en artículos que no tienen ese enfoque predominante, como se ha podido corroborar en la lectura detallada de los trabajos. La mayoría de las investigaciones analizadas aportan aplicaciones tecnológicas (32\%), aportaciones metodológicas $(31 \%)$ y estudios de casos $(25 \%)$. Investigaciones futuras encaminadas al desarrollo teórico-conceptual y análisis crítico del ST, completarán la brecha existente entre el conocimiento y desarrollo operativo del ST.

El enfoque de investigación más utilizado es el de Smart Experience $(36,4 \%)$ y Smart Destinations (29\%). La aplicación de la tecnología para la mejora de la experiencia turística se establece como un elemento clave del ST(Buhalis y Amaranggana, 2015; Gretzel, 2016; Gretzel y Jamal, 2009; Neuhofer, Buhalis, y Ladkin, 2012; 2015). En este sentido, investigaciones futuras que identifiquen cada una de estas smart technologies, así como su ámbito de aplicación en los destinos inteligentes y sus aspectos de mejora más relevantes, pueden favorecer el desarrollo de estos destinos.

Las temáticas de interés se decantan mayoritariamente hacia los "Sistemas de Información, plataformas tecnológicas y ciencia de los datos" (42\%) y el "Marketing Digital" $(30,2 \%)$. Son dos categorías muy amplias que pueden dar lugar al desarrollo de investigaciones más específicas que analicen, por ejemplo, la ciencia de los datos y su relación con la mejora de los procesos de gestión en los destinos inteligentes. Del mismo modo, investigaciones de aplicación de tecnología enfocadas a la mejora de los procesos de gobernanza (Pulido y Pulido, 2014), aspecto clave de los destinos inteligentes, pueden aportar conocimiento y establecer procesos mejora de la administración. Como por ejemplo los Datos Abiertos desde una perspectiva del destino turístico inteligente. 
Respecto a la procedencia geográfica de las investigaciones, y centrados ahora en el ámbito nacional, contrasta el liderazgo institucional de España en el desarrollo de destinos turísticos inteligentes y la reducida producción científica asociada. Se constata así una brecha entre el conocimiento teórico-conceptual y el desarrollo práctico que debe cubrir la academia para orientar una correcta aplicación del enfoque destinos inteligentes.

Las tecnologías que más se asocian al ST son el Internet de las Cosas (39\%), el Big Data (29\%) y la Realidad Aumentada (9\%). Representan el 77\% de las tecnologías mencionadas. El análisis de los usos concretos de cada una de ellas es otra de los aspectos de interés respecto a investigaciones futuras. A este respecto, investigaciones que analicen en profundidad estas aplicaciones mediante técnicas de SLR, pueden aportar información de mucho interés y facilitar su desarrollo.

Esta investigación se configura como una aproximación inicial a los estudios de mapeo sistemático aplicados al turismo. No obstante, presenta una serie de limitaciones que se han de señalar. La primera, y quizá más importante, es que esta técnica no profundiza en el contenido de los trabajos, sino que los clasifica de acuerdo a categorías para obtener una aproximación general sobre la producción científica en un determinado campo de estudio. No siempre es sencillo clasificar los artículos en una u otra categoría, por lo que entraña cierto grado de subjetividad. Por ese motivo se realiza una puesta en común entre los investigadores antes de la clasificación final. La estrategia de búsqueda es otro aspecto que puede aportar limitaciones, desde la selección de la etiqueta hasta el motor científico donde se realiza la búsqueda. Este trabajo se establece como un punto de partida para futuros estudios de mapeo sistemático en turismo.

\section{REFERENCIAS BIBLIOGRÁFICAS}

ALBACETE SÁEZ, C.A., FUENTES FUENTES, M. y CARMEN HARO-DOMÍNGUEZ, M. (2013): «La investigación española en turismo con impacto internacional (19972011). Una perspectiva desde la economía y la dirección de la empresa», Cuadernos de Economia y Direccion de la Empresa, 16 (1), pp. 17-28. http://doi.org/10.1016/j. cede.2012.05.003

ALBINO, V., BERARDI, U. y DANGELICO, R.M. (2015): «Smart Cities: Definitions, Dimensions, Performance, and Initiatives», Journal of Urban Technology, 22 (1), pp. 3-21. http://doi.org/10.1080/10630732.2014.942092

ARCHAMBAULT, É., CAMPBELL, D., GINGRAS, Y. y LARIVIÈRE, V. (2009): «Comparing bibliometric statistics obtained from the web of science and Scopus», Journal of the American Society for Information Science and Technology, 60 (7), pp. 1320-1326. http://doi.org/10.1002/asi.21062

BAGGIO, R. y COOPER, C. (2015): «Knowledge transfer in a tourism destination: the effects of a network structure», The Service Industries Journal, (4), pp. 145-150. http:// doi.org/10.1016/j.jdmm.2015.02.001

BAR-ILAN, J. (2010): «Citations to the "Introduction to informetrics" indexed by WOS, Scopus and Google Scholar», Scientometrics, 82(3), pp. 495-506. http://doi. org/10.1007/s11192-010-0185-9 
BENCKENDORFF, P. (2009): «Themes and trends in Australian and New Zealand tourism research: A social network analysis of citations in two leading journals (19942007)», Journal of Hospitality and Tourism Management, 16 (1), pp. 1-15. http://doi. org/10.1375/jhtm.16.1.1

BENCKENDORFF, P. y ZEHRER, A. (2013): "A network analysis of tourism research», Annals of Tourism Research, 43, pp. 121-149. http://doi.org/10.1016/j. annals.2013.04.005

BUHALIS, D. (2011): «E-Tourism», Contemporary Tourism Reviews, pp. 1-38. [en línea]. Dis ponible en: http://www.goodfellowpublishers.com/free_files/Contemporary-Tourism-Review-Etourism-66769a7ed0935d0765318203b843a64d.pdf. [Acceso 9 de enero de 2018]

BUHALIS, D. y AMARANGGANA, A. (2015): «Smart Tourism Destinations Enhancing Tourism Experience Through Personalisation of Services», en Information and Communication Technologies in Tourism 2015 (Vol. 28, pp. 377-389). http://doi. org/10.1007/978-3-319-14343-9_28

BUHALIS, D. y FOERSTE, M. (2014): «SoCoMo marketing for travel and tourism: Empowering co-creation of value», Journal of Destination Marketing and Management, 4 (3), pp. 151-161. http://doi.org/10.1016/j.jdmm.2015.04.001

BUHALIS, D. y LAW, R. (2008): «Progress in information technology and tourism management: 20 years on and 10 years after the Internet-The state of eTourism research», Tourism Management, 29 (4), pp. 609-623. http://doi.org/10.1016/j.tourman.2008.01.005

BUHALIS, D. y LICATA, M. C. (2002): «The future eTourism intermediaries», Tourism Management, 23(3), pp. 207-220. http://doi.org/10.1016/S0261-5177(01)00085-1

BUHALIS, D., WAGNER, R. y KINGDOM, U. (2013): «E-destinations: Global Best Practice in Tourism Technologies and Applications», Information and Communication Technologies in Tourism 2013, pp. 119-130. http://doi.org/10.1007/978-3-642-36309-2

CARAGLIU, A., DEL BO, C. y NIJKAMP, P. (2011): «Smart Cities in Europe», Journal of Urban Technology, 18(2), pp. 65-82. http://doi.org/10.1080/10630732.2011.601117

CHADEGANI, A.A., SALEHI, H., YUNUS, M., FARHADI, H., FOOLADI, M. y FARHADI, M. (2013): «A Comparison between Two Main Academic Literature Collections : Web of Science and Scopus Databases», Asian Social Science, 9(5), pp. 18-26. http://doi.org/10.5539/ass.v9n5p18

CHUNG, N. y KOO, C. (2015): «The use of social media in travel information search», Telematics and Informatics, 32(2), pp. 215-229. http://doi.org/10.1016/j.tele.2014.08.005

CHUNG, N., LEE, H., LEE, S.J. y KOO, C. (2015): «The influence of tourism website on tourists' behavior to determine destination selection: A case study of creative economy in Korea», Technological Forecasting and Social Change, 96, pp. 130-143. http://doi. org/10.1016/j.techfore.2015.03.004

CORRAL-MARFIL, J.A., y CÁNOVES VALIENTE, G. (2014): «La colaboración científica en turismo: grupos y redes de investigación en Cataluña», Documents d'Anàlisi Geogràfica, vol. 60/1, pp. 57-86.

DATTA, A. (2015): «A 100 smart cities, a 100 utopias», Dialogues in Human Geography, 5(1), pp. 49-53. http://doi.org/10.1177/2043820614565750 
DEL CHIAPPA, G., y BAGGIO, R. (2015): «Knowledge transfer in smart tourism destinations: Analyzing the effects of a network structure», Journal of Destination Marketing y Management, 4 (3), pp. 145-150. http://doi.org/10.1016/j.jdmm.2015.02.001

FALAGAS, M.E., PITSOUNI, E.I., MALIETZIS, G.A. y PAPPAS, G. (2007): «Comparison of PubMed, Scopus, Web of Science, and Google Scholar: strengths and weaknesses», The FASEB Journal, 22 (2), pp. 338-342. http://doi.org/10.1096/fj.07-9492LSF

FERNÁNDEZ-GÜELL, J.M. (2015): «Ciudades Inteligentes. La mitificación de las nuevas tecnologías como respuesta a los retos de las ciudades contemporáneas», Economía Industrial, pp.17-28.

FERNÁNDEZ GONZÁLEZ, M. (2015): La Smart City como imaginario socio-tecnológico digital. La construcción de la utopía urbana digital (Tesis doctoral). Universidad del País Vasco.

FERNÁNDEZ V., PÉREZ F., MONZÓN A. y TORREGROSA A. (2015): «Buenas prácticas en Ciudades Inteligentes. Respondiendo a los retos urbanos». Comunicación presentada en el I Congreso de Ciudades Inteligentes. Madrid 24-25 marzo.

GIFFINGER, R. (2007): Smart cities Ranking of European medium-sized cities. October (Vol. 16). [en línea]. Disponible en : http://linkinghub.elsevier.com/retrieve/pii/ S026427519800050X [Acceso 18 de diciembre de 2017]

GOODMAN, D. y DEIS, L. (2005): «Web of Science ( 2004 version ) and Scopus Composite Score: Scopus Reviewed by: David Goodman INTENDED AUDIENCE», The Charleston Advisor, (January), pp. 5-21.

GOODMAN, D. y DEIS, L. (2007): «Update on Scopus and Web of Science», The Charleston Advisor, (January), pp. 15-18.

GRAZIANO, T. (2014): «Boosting Innovation and Development? the Italian Smart Tourism: a Critical Perspective», European Journal of Geography, 5 (4), pp. 6-18.

GRETZEL, U. (2011): «Intelligent systems in tourism», Annals of Tourism Research, 38 (3), pp. 757-779. http://doi.org/10.1016/j.annals.2011.04.014

GRETZEL, U. (2016): «Creating Experience Value in Tourism», Annals of Tourism Research, 57 (2016), pp. 285-297. http://doi.org/10.1016/j.annals.2015.12.008

GRETZEL, U., y JAMAL, T. (2009): «Conceptualizing the Creative Tourist Class: Technology, Mobility, and Tourism Experiences», Tourism Analysis, 14 (979), pp. 471-481. http://doi.org/10.3727/108354209X12596287114219

GRETZEL, U., SIGALA, M., XIANG,Z. y KOO, C. (2015): «Smart tourism : foundations and developments», Electron Markets, 25 (3), pp. 179-188. https://doi.org/10.1007/ s12525-015-0196-8

GRETZEL, U., WERTHNER, H., KOO, C. y LAMSFUS, C. (2015): «Conceptual foundations for understanding smart tourism ecosystems», Computers in Human Behavior, 50, pp. 558-563. http://doi.org/10.1016/j.chb.2015.03.043

GUO, Y., LIU, H. y CHAI, Y. (2014): «The embedding convergence of smart cities and tourism internet of things in China: An advance perspective», Advances in Hospitality and Tourism Research, 2 (1), pp. 54-69.

HJALAGER, A.M. (2013): «100 Innovations That Transformed Tourism», Journal of Travel Research, 54 (1), pp. 3-21. http://doi.org/10.1177/0047287513516390 
HUANG, C. D., GOO, J., NAM, K. y WOO, C. (2017): «Information y Management Smart tourism technologies in travel planning: The role of exploration and exploitation», Information y Management, 54 (6), pp. 757-770. http://doi.org/10.1016/j. im.2016.11.010

HUNTER, W.C., CHUNG, N., GRETZEL, U. y KOO, C. (2015): «Constructivist Research in Smart Tourism», Asia Pacific Journal of Information Systems, 25 (1), pp. 105-120. http://doi.org/10.14329/apjis.2015.25.1.105

IBM. (2010): Smarter cities for smarter growth. How cities can optimize their systems for the talent-based economy. IBM Global Business Services. New York. [en línea]. Disponible en: http://www-935.ibm.com/services/us/gbs/bus/html/smarter-cities.html [Acceso 29 de diciembre de 2017]

IVARS-BAIDAL, J.A., CELDRÁN-BERNABEU, M. A., MAZÓN, J.N. y PERLESIVARS, Á. F. (2017): «Smart destinations and the evolution of ICTs: a new scenario for destination management?», Current Issues in Tourism, 3500(October), pp. 1-20. http://doi.org/10.1080/13683500.2017.1388771

IVARS-BAIDAL, J.A., CELDRÁN-BERNABEU, M.A., y VERA-REBOLLO, J.F. (2017): «Orientaciones prácticas para el diseño de productos turísticos en el ámbito local», en Las claves del turismo en la Comunitat Valenciana: orientaciones para la toma de decisiones. Valencia, Universidad de Valencia, pp. 65-79.

IVARS-BAIDAL, J.A., SOLSONA MONZONÍS, F.J. y GINER SÁNCHEZ, D. (2016): «Gestión turística y tecnologías de la información y la comunicación (TIC): El nuevo enfoque de los destinos inteligentes», Documents d'Anàlisi Geogràfica, 62 (2), pp. 327-346.

JACOBSEN, J.K.S. y MUNAR, A.M. (2012): «Tourist information search and destination choice in a digital age», Tourism Management Perspectives, 1 (1), pp. 39-47. http:// doi.org/10.1016/j.tmp.2011.12.005

KITCHENHAM, B. (2010): «What's up with software metrics? - A preliminary mapping study», Journal of Systems and Software, 83 (1), pp. 37-51. http://doi.org/10.1016/j. jss.2009.06.041

KITCHENHAM, B.A., BUDGEN, D. y BRERETON, O. (2011): «Using mapping studies as the basis for further research - A participant-observer case study», Information and Software Technology, 53 (6), pp. 638-651. http://doi.org/10.1016/j.infsof.2010.12.011

KITCHIN, R. (2014): «Making sense of smart cities: addressing present shortcomings», Cambridge Journal of Regions, Economy and Society, 8 (1), pp. 131-136. http://doi. org/10.1093/cjres/rsu027

KOMNINOS, N., SCHAFFERS, H. y PALLOT, M. (2011): «Developing a Policy Roadmap for Smart Cities and the Future Internet», eChallenges e2011, 1-8. http://doi. org/10.1109/MCOM.2013.6525605

KOMNINOS, N. y TSARCHOPOULOS, P. (2013): «Toward Intelligent Thessaloniki: From an Agglomeration of Apps to Smart Districts», Journal of the Knowledge Economy, 4 (2), pp. 149-168. http://doi.org/10.1007/s13132-012-0085-8

KOO, C., SHIN, S., GRETZEL, U., HUNTER, W.C. y CHUNG, N. (2016): «Conceptualization of Smart Tourism Destination Competitiveness», Asia Pacific Journal of Information Systems, 26(4), pp. 561-576. http://doi.org/10.14329/apjis.2016.26.4.561 
KOSEOGLU, M.A., RAHIMI, R., OKUMUS, F., y LIU, J. (2016): «Bibliometric studies in tourism», Annals of Tourism Research, 61, pp. 180-198. http://doi.org/10.1016/j. annals.2016.10.006

LI, Y., HU, C., HUANG, C. y DUAN, L. (2016): «The concept of smart tourism in the context of tourism information services», Tourism Management, 58, pp. 293-300. https://doi.org/10.1016/j.tourman.2016.03.014.

LÓPEZ DE AVILA MUÑOZ, A. y GARCÍA SÁNCHEZ, S. (2015): «Destinos turísticos inteligentes», Economía Industrial, no 395, pp. 61-69

LUQUE GIL, A.M., ZAYAS FERNÁNDEZ, B. y CARO HERRERO, J.L. (2015): «Los Destinos Turísticos Inteligentes en el marco de la Inteligencia Territorial: conflictos y oportunidades», Investigaciones Turísticas, nº10, pp.1-25.

MARCH, H. y RIBERA-FUMAZ, R. (2014): «Smart contradictions: The politics of making Barcelona a Self-sufficient city», European Urban and Regional Studies, pp. 1-15. http://doi.org/10.1177/0969776414554488

MCKERCHER, B. (2008): «A citation analysis of tourism scholars», Tourism Management, 29 (6), pp. 1226-1232. http://doi.org/10.1016/j.tourman.2008.03.003

MICHAEL HALL, C. (2011): «Publish and perish? Bibliometric analysis, journal ranking and the assessment of research quality in tourism», Tourism Management, 32 (1), pp. 16-27. http://doi.org/10.1016/j.tourman.2010.07.001

MORENO ALONSO, C. (2015): Desarrollo de un modelo de evaluación de ciudades basado en el concepto de Ciudad Inteligente (Smart City) (Tesis doctoral). Universidad Politécnica de Madrid.

MUNAR, A.M. y JACOBSEN, J.K.S. (2014): «Motivations for sharing tourism experiences through social media», Tourism Management, 43, pp. 46-54. http://doi. org/10.1016/j.tourman.2014.01.012

NACIONES UNIDAS (2014): E-Government Survey 2014. E-Government for the future we want. [en línea]. Disponible en: https://publicadministration.un.org/egovkb/portals/egovkb/documents/un/2014-survey/e-gov_complete_survey-2014.pdf. [Acceso 27 diciembre 2017]

NAM, T. y PARDO, T.A. (2011): «Conceptualizing Smart City with Dimensions of Technology , People and Institutions», The Proceedings of the 12th Annual International Conference on Digital Government Research, pp. 282-291.http://doi. org/10.1145/2037556.2037602

NAPOLEÃO, B. M., FELIZARDO, K.R., DE SOUZA, É.F. y VIJAYKUMAR, N.L. (2017): «Practical similarities and differences between Systematic Literature Reviews and Systematic Mappings: a tertiary study», The 29th International Conference on Software Engineering and Knowledge Engineering. http://doi.org/10.18293/SEKE2017069

NEUHOFER, B., BUHALIS, D. y LADKIN, A. (2012): «Conceptualising technology enhanced destination experiences», Journal of Destination Marketing and Management, 1 (1-2), pp. 36-46. http://doi.org/10.1016/j.jdmm.2012.08.001

NEUHOFER, B., BUHALIS, D. y LADKIN, A. (2015). «Smart technologies for personalized experiences: a case study in the hospitality domain», Electronic Markets, 25 (3), pp. 243-254. http://doi.org/10.1007/s12525-015-0182-1 
ONU-HABITAT (2016): Reporte Ciudades del Mundo 2016. Programa de las Naciones Unidas para los Asentamientos Humanos (ONU-Habitat). [en línea]. Disponible en: http://nua.unhabitat.org/uploads/Reportedelasciudades2016.pdf. [Acceso 14 diciembre 2017]

PARRA-LÓPEZ, E., BULCHAND-GIDUMAL, J., GUTIÉRREZ-TAÑO, D. y DÍAZARMAS, R (2011): «Intentions to use social media in organizing and taking vacation trips», Computers in Human Behavior, 27 (2), pp. 640-654. https://doi.org/10.1016/j. chb.2010.05.022.

PETERSEN, K., FELDT, R., MUJTABA, S., y MATTSSON, M. (2008): «Systematic Mapping Studies in Software Engineering». 12Th International Conference on Evaluation and Assessment in Software Engineering, 17, 10. http://doi.org/10.1142/ S0218194007003112

PETERSEN, K., VAKKALANKA, S. y KUZNIARZ, L. (2015): «Guidelines for conducting systematic mapping studies in software engineering: An update», Information and Software Technology, 64, pp. 1-18. http://doi.org/10.1016/j.infsof.2015.03.007

PICKERING, C. y BYRNE, J. (2014): «The benefits of publishing systematic quantitative literature reviews for $\mathrm{PhD}$ candidates and other early-career researchers», Higher Education Research and Development, 33 (3), pp. 534-548. http://doi.org/10.1080/0 7294360.2013 .841651

PICKERING, C., ROSSI, S.D., HERNANDO, A. y BARROS, A. (2018): «Current knowledge and future research directions for the monitoring and management of visitors in recreational and protected areas», Journal of Outdoor Recreation and Tourism, 21(June 2017), pp. 10-18. http://doi.org/10.1016/j.jort.2017.11.002

PIERCE, P., RICCIARDI, F. y ZARDINI, A. (2017): «Smart cities as organizational fields: A framework for mapping sustainability-enabling configurations», Sustainability (Switzerland), 9 (9), pp. 1-21. http://doi.org/10.3390/su9091506

PULIDO, M.C. y PULIDO, J.I. (2014): «¿Existe gobernanza en la actual gestión de los destinos turísticos? Estudio de casos", Pasos, vol. 12 (4), pp. 685-705.

PWC, S. P. DE, \& SCHOOL, I. B. (2015): Smart cities. La transformación digital de las ciudades. [en línea]. Disponible en: https://iot.telefonica.com/libroblanco-smart-cities/ media/libro-blanco-smart-cities-esp-2015.pdf. [Acceso 22 diciembre 2015]

SEGITTUR (2015): Libro blanco destinos turísticos inteligentes: construyendo el futuro. [en línea]. Disponible en: http://www.segittur.es/es/DTI/dti-detalle/Libro-Blanco-Destinos-Tursticos-Inteligentes-/\#.Wmy0EZOdVsM. [Acceso 27 diciembre 2017]

SHELTON, T., ZOOK, M. y WIIG, A. (2015): «The actually existing smart city», Cambridge Journal of Regions Economy and Society, 8 (1), pp. 13-25. http://doi.org/DOI $10.1093 / \mathrm{cjres} / \mathrm{rsu} 026$

SINGH, B. (2015): «Smart city-smart life - Dubai Expo 2020», Middle East Journal of Business, 10 (4), pp. 49-52.[en línea] Disponible en: http://search.ebscohost.com/ login. aspx ?direct=trueydb=bthy $\mathrm{AN}=110343622 \mathrm{ylang}=$ esysite=ehost-liveyscope $=$ site . [Acceso 19 diciembre 2017]

SMART TOURISM RESARCH CENTER (2017): Research topics. [en línea] Disponible en: http://strc.khu.ac.kr/contents/bbs/bbs_content.html?bbs_cls_cd=003002002. [Acceso 27 diciembre 2017] 
SUN, Y., SONG, H., JARA, A. y BIE, R. (2016): «Big Data Analytics on Smart and Connected Communities Using Internet of Things», International Journal of Pharmacy y Technology, 8 (4), pp. 19590-19601.

TRIBE, J. y MKONO, M. (2017): «Not such smart tourism? The concept of e-lienation», Annals of Tourism Research, 66, pp. 105-115. http://doi.org/10.1016/j. annals.2017.07.001

VANOLO, A. (2014): «Smartmentality: The Smart City as Disciplinary Strategy», Urban Studies, 51 (5), pp. 883-898. http://doi.org/10.1177/0042098013494427

VIEIRA, E.S. y GOMES, J.A.N.F. (2009): «A comparison of Scopus and Web of science for a typical university. Scientometrics, 81 (2), 587-600. http://doi.org/10.1007/s11192009-2178-0

WANG, D., LI, X. y LI, Y. (2013): «China's “smart tourism destination” initiative: A taste of the service-dominant logic», Journal of Destination Marketing and Management, 2 (2), pp. 59-61. http://doi.org/10.1016/j.jdmm.2013.05.004

WEBSTER, J. y WATSON, R.T. (2002): «Analyzing the past to prepare for the future: writing a literature review», MIS Quarterly, vol. 26 (2), pp. xiii-xxiii.

XIANG, Z., WANG, D., O'LEARY, J.T. y FESENMAIER, D.R. (2015): «Adapting to the Internet: Trends in Travelers' Use of the Web for Trip Planning», Journal of Travel Research, 54(4), pp. 511-527. http://doi.org/10.1177/0047287514522883

XIANG, Z., MAGNINI, V.P. y FESENMAIER, D.R. (2015): «Information technology and consumer behavior in travel and tourism: Insights from travel planning using the internet», Journal of Retailing and Consumer Services, 22, pp. 244-249. http://doi. org/10.1016/j.jretconser.2014.08.005

XIANG, Z., TUSSYADIAH, I. y BUHALIS, D. (2015): «Smart destinations: Foundations, analytics, and applications», Journal of Destination Marketing and Management, 4 (3). pp. 143-144. http://doi.org/10.1016/j.jdmm.2015.07.001

XIAO, H. y SMITH, S.L.J. (2007): «The use of Tourism knowledge. Research Propositions», Annals of Tourism Research, 34 (2), pp. 310-331. http://doi.org/10.1016/j. annals.2006.09.001

YANG, E.C.L., KHOO-LATTIMORE, C. y ARCODIA, C. (2017): «A systematic literature review of risk and gender research in tourism», Tourism Management, 58, 89-100. http://doi.org/10.1016/j.tourman.2016.10.011 